\title{
Advanced imaging of colorectal cancer: From anatomy to molecular imaging
}

\author{
Roberto García-Figueiras ${ }^{1}$ - Sandra Baleato-González ${ }^{1}$ Anwar R. Padhani ${ }^{2}$. \\ Ana Marhuenda ${ }^{3}$ - Antonio Luna ${ }^{4,5}$ - Lidia Alcalá ${ }^{4}$ - Ana Carballo-Castro ${ }^{6}$. \\ Ana Álvarez-Castro ${ }^{7}$
}

Received: 12 August 2015 /Revised: 30 December 2015 / Accepted: 19 January 2016/Published online: 30 April 2016

(C) The Author(s) 2016. This article is published with open access at Springerlink.com

\begin{abstract}
Imaging techniques play a key role in the management of patients with colorectal cancer. The introduction of new advanced anatomical, functional, and molecular imaging techniques may improve the assessment of diagnosis, prognosis, planning therapy, and assessment of response to treatment of these patients. Functional and molecular imaging techniques in clinical practice may allow the assessment of tumourspecific characteristics and tumour heterogeneity. This paper will review recent developments in imaging technologies and the evolving roles for these techniques in colorectal cancer.
\end{abstract}

\section{Teaching Points}

- Imaging techniques play a key role in the management of patients with colorectal cancer.
- Advanced imaging techniques improve the evaluation of these patients.

- Functional and molecular imaging allows assessment of tumour hallmarks and tumour heterogeneity.

Keywords Colorectal neoplasms $\cdot$ MRI, functional · Perfusion imaging $\cdot$ Molecular imaging $\cdot$ Multimodal imaging

Abbreviations
$\begin{array}{ll}\text { (3D) } & \text { 3-dimensional } \\ \text { (ADC) } & \text { Apparent diffusion coefficient } \\ \text { (AUC) } & \text { Area under the curve } \\ \text { (BF) } & \text { Blood flow } \\ \text { (BV) } & \text { Blood volume }\end{array}$

1 Department of Radiology, Hospital Clínico Universitario de Santiago de Compostela, Choupana s/n, 15706 Santiago de Compostela, Spain

Sandra Baleato-González

baleatorum@hotmail.com

Anwar R. Padhani

anwar.padhani@stricklandscanner.org.uk

Ana Marhuenda

amarflu@gmail.com

Antonio Luna

aluna70@htime.org

Lidia Alcalá

1.alcala.o@htime.org

Ana Carballo-Castro

ana.maria.carballo.castro@sergas.es

Ana Álvarez-Castro

anacastro189@gmail.com
2 Paul Strickland Scanner Centre, Mount Vernon Cancer Centre, Northwood, Middlesex, England HA6 2RN, UK

3 Department of Radiology, IVO (Instituto Valenciano de Oncología), C/ Beltrán Báguena, 8, 46009 Valencia, Spain

4 Department of Radiology, Advanced Medical Imaging, Clinica Las Nieves, SERCOSA, Grupo Health Time, C/ Carmelo Torres 2, 23007 Jaén, Spain

5 Case Western Reserve University, Cleveland, OH, USA Santiago de Compostela, Choupana s/n, 15706 Santiago de Compostela, Spain

7 Department of Gastroenterology, Colorectal Cancer Group, Hospital Clínico Universitario de Santiago de Compostela, Choupana s/n, Santiago de Compostela 15706, Spain
6 Department of Radiotherapy, Hospital Clínico Universitario de 


\begin{tabular}{|c|c|c|}
\hline (BOLD) & Blood oxygenation level dependent & management and prognosis and have decreased CRC mortal- \\
\hline (CTP) & Chemotherapy & ity rate by $20 \%$ in the last years [2]. \\
\hline (CRTP) & Chemoradiotherapy & Conventional imaging techniques have clear limitations for \\
\hline (Cho) & Choline & the evaluation of important tumour features. For example, 9- \\
\hline$(\mathrm{CRC})$ & Colorectal cancer & $10 \%$ of patients with computed tomography $(\mathrm{CT})$-indetermi- \\
\hline$(\mathrm{pCR})$ & Complete pathologic response & nate lung and/or liver lesions during radiological staging of \\
\hline (CT) & Computed tomography & $\mathrm{CRC}$ had definite metastases $[3,4]$. Besides, an increasing \\
\hline (CTC) & Computed tomographic colonography & importance is being placed on the non-invasive imaging as- \\
\hline (DW) & Diffusion-weighted & sessment of tumour-specific characteristics [5-8]. Functional \\
\hline (DWI) & Diffusion-weighted imaging & and molecular imaging (FMI) techniques have emerged to \\
\hline ECT) & Dual-energy computed tomography & address these limitations. This paper is focused on the current \\
\hline (DCE) & Dynamic contrast-enhanced & role of advanced imaging modalities in CRC patient \\
\hline (EES) & Extravascular extracellular space & management. \\
\hline
\end{tabular}

$\left({ }^{18} \mathrm{~F}-\mathrm{FDG}\right) \quad$ Fluorodeoxyglucose

(FLT) $\quad{ }^{18} \mathrm{~F}$-3-deoxy-3-fluorothymidine

(FMI) Functional and molecular imaging

(IVIM) Intravoxel incoherent motion

(IC) Iodine concentration

(Lip) Lipids

(LNs) Lymph nodes

(MRI) Magnetic resonance imaging

(MRL) MR lymphography

(MRS) MR spectroscopy

(MTT) Mean transit time

(NPV) Negative predict value

(OS) Overall survival

(PCT) Perfusion CT

(f) Perfusion fraction

(D) Perfusion-free diffusion

(PP) Portal phase

(PPV) Positive predict value

$\left({ }^{1} \mathrm{H}\right) \quad$ Proton

$\left(\mathrm{k}_{\mathrm{ep}}\right) \quad$ Rate constant

(PET) Positron emission tomography

(RC) Rectal cancer

(SUV) Standardized uptake values

(Krans $\quad$ Transfer constant

(TVRR) Tumour volume reduction rate

(USPIO) Ultrasmall iron oxide particles

(VEGF) Vascular endothelial growth factor

(WB)-DWI Whole-body diffusion-weighted imaging

\section{Introduction}

Colorectal cancer (CRC) represents one of the most commonly diagnosed cancers worldwide. It is the second most common cause of cancer death in the western world [1]. A multidisciplinary approach to CRC management, which includes the radiologist's role, and the optimization of screening, biomarker and genomic analysis, imaging evaluation, surgical techniques, and therapies have improved patients' management and prognosis and have decreased CRC mortaly rate by $20 \%$ in the last years [2].

Conventional imaging techniques have clear limitations for the evaluation of important tumour features. For example, 9$10 \%$ of patients with computed tomography (CT)-indeterminate lung and/or liver lesions during radiological staging of CRC had definite metastases [3, 4]. Besides, an increasing importance is being placed on the non-invasive imaging assessment of tumour-specific characteristics [5-8]. Functional and molecular imaging (FMI) techniques have emerged to role of advanced imaging modalities in CRC patient management.

\section{Anatomical imaging techniques in CRC}

\section{Conventional imaging technique}

Conventional imaging techniques play a central role in CRC because they depict relationships of the tumour to surgical landmarks (e.g., the circumferential resection margin in the rectum), the presence of important prognostic features, evaluate tumour response to treatment, and are useful for surveillance after therapy. In the case of rectal cancer (RC), magnetic resonance imaging (MRI) is the best imaging technique for evaluating main factors that affect treatment and prognosis, including tumour length, location from the anal verge, relationship to the peritoneal reflection, T-stage, depth of extramural tumour growth, lymph node (LNs) status, vascular and neural invasion, distance to the mesorectal resection margin, and invasion to adjacent structures $[8,9]$. Beside this, the main focus for innovations in medical imaging has been the achievement of excellence in anatomical resolution. To date, imaging techniques allow image segmentation and volumetric model reconstruction with different clinical applications in CRC.

\section{Computed tomographic colonography}

Computed tomographic colonography (CTC) involves the use of a CT scanner to produce 2- and 3-dimensional (3D) images of the entire colon and rectum obtained after air insufflation (Fig. 1). CTC can be considered the best radiological diagnostic test for screening CRC and polyps. It has been established that its diagnostic performance for the detection of CRC is similar to that of conventional colonoscopy and is clearly superior to that of a barium enema [10]. Besides, CTC is less invasive than a conventional colonoscopy and easy to perform. Different indications have emerged supported by strong evidence-based data and scientific societies including (1) incomplete, failed, or unfeasible conventional colonoscopy (for diagnosing synchronous cancers), (2) elderly and frail patients (who are more likely to have a complicated colonoscopy), (3) 


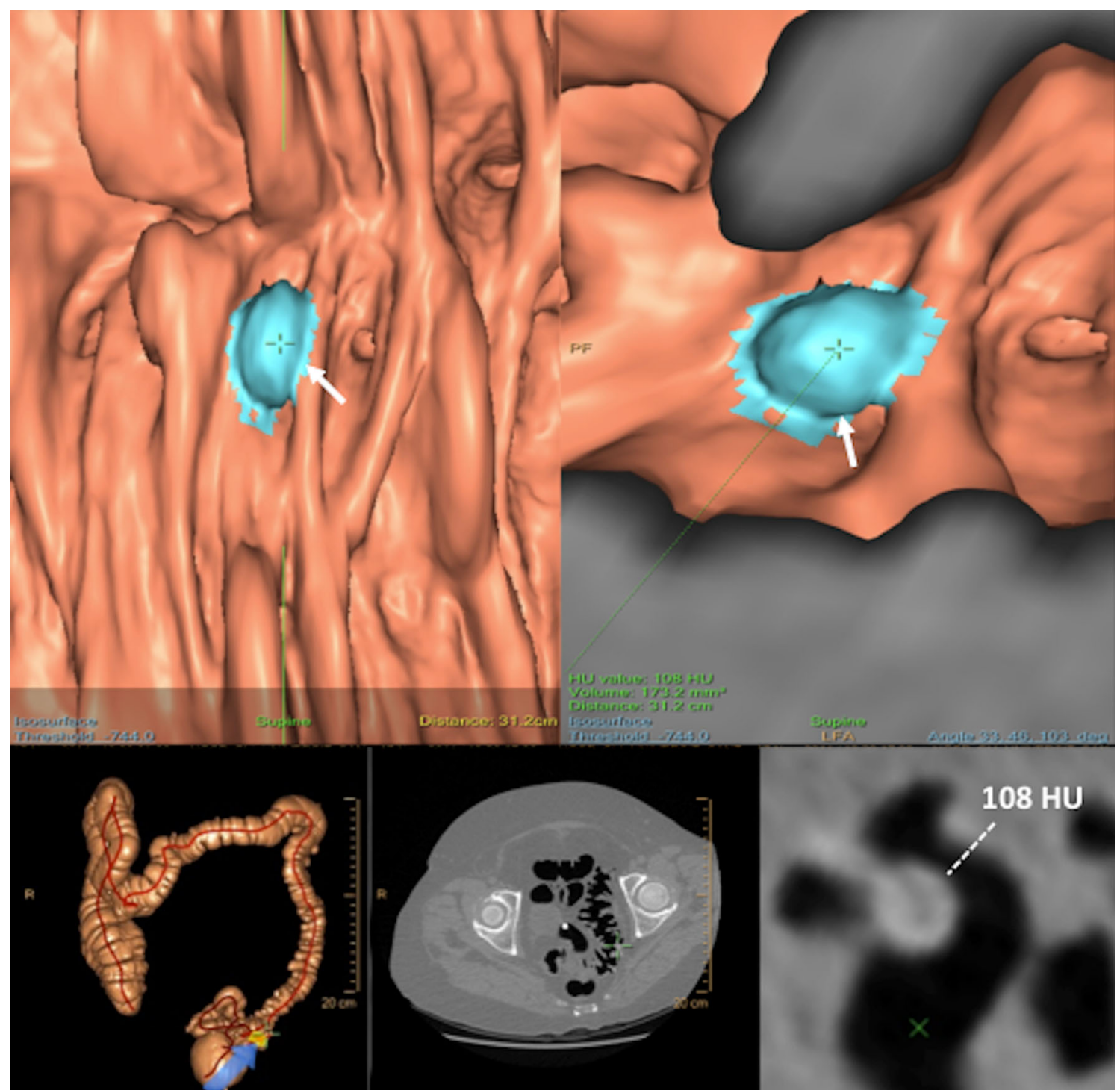

Fig. 1 CT colonography in a 40-year-old woman with rectal bleeding. $3 \mathrm{D}$ endoluminal virtual dissection view (presenting the bowel as if it has been straightened and unfolded) (top left) and 3D endoluminal view of the colon (top right) showed a suspicious finding (arrows) for the

evaluation of alarm symptoms suggestive of CRC, (4) tumour localization (especially for laparoscopic surgery), (5) and evaluation of diverticular disease and of patients with colonic stoma [11]. Other indications, many of which are still being debated, include CRC screening and surveillance after surgery for CRC or polypectomy [11-13].

\section{Volumetry in $C R C$}

Tumour sizes and volumes have been proven to be an important prognostic indicator for a variety of tumours. However, these features were not found useful in the TNM staging system in CRC and in predicting the clinical outcome of patients, though published papers are sometimes contradictory [14-16]. Simple methods are available for measuring volume based on computer-aided polyp detection system (blue lesion). Correlation of these $3 \mathrm{D}$ endoluminal views with traditional images at CT demonstrated that this finding corresponded to a high-density (108 HU) impacted diverticulum

different semi-automated techniques. In the case of RC, tumour volume reduction rate (TVRR) following chemoradiotherapy (CRTP) based on T2-weighted volumetry may have a predictive value. TVRR shows a significant correlation with tumour pathological regression grade after preoperative CRTP [17] and a volume reduction ratio $>75 \%$ is associated with an increased pathologic complete response rate [18]. The main limitation of $\mathrm{T} 2$-weighted images in the restaging of $\mathrm{RC}$ post-CRTP is its inability to distinguish between small remaining tumour foci and fibrosis, which impacts negatively on its sensitivity. Moreover, it is difficult to decide which areas remain suspicious for tumours on $\mathrm{T} 2$-weighted images and should be included in the volume measurements. Tumour volumetry based on the signal-intensity characteristics of dynamic contrast-enhanced (DCE) or diffusion-weighted (DW) 


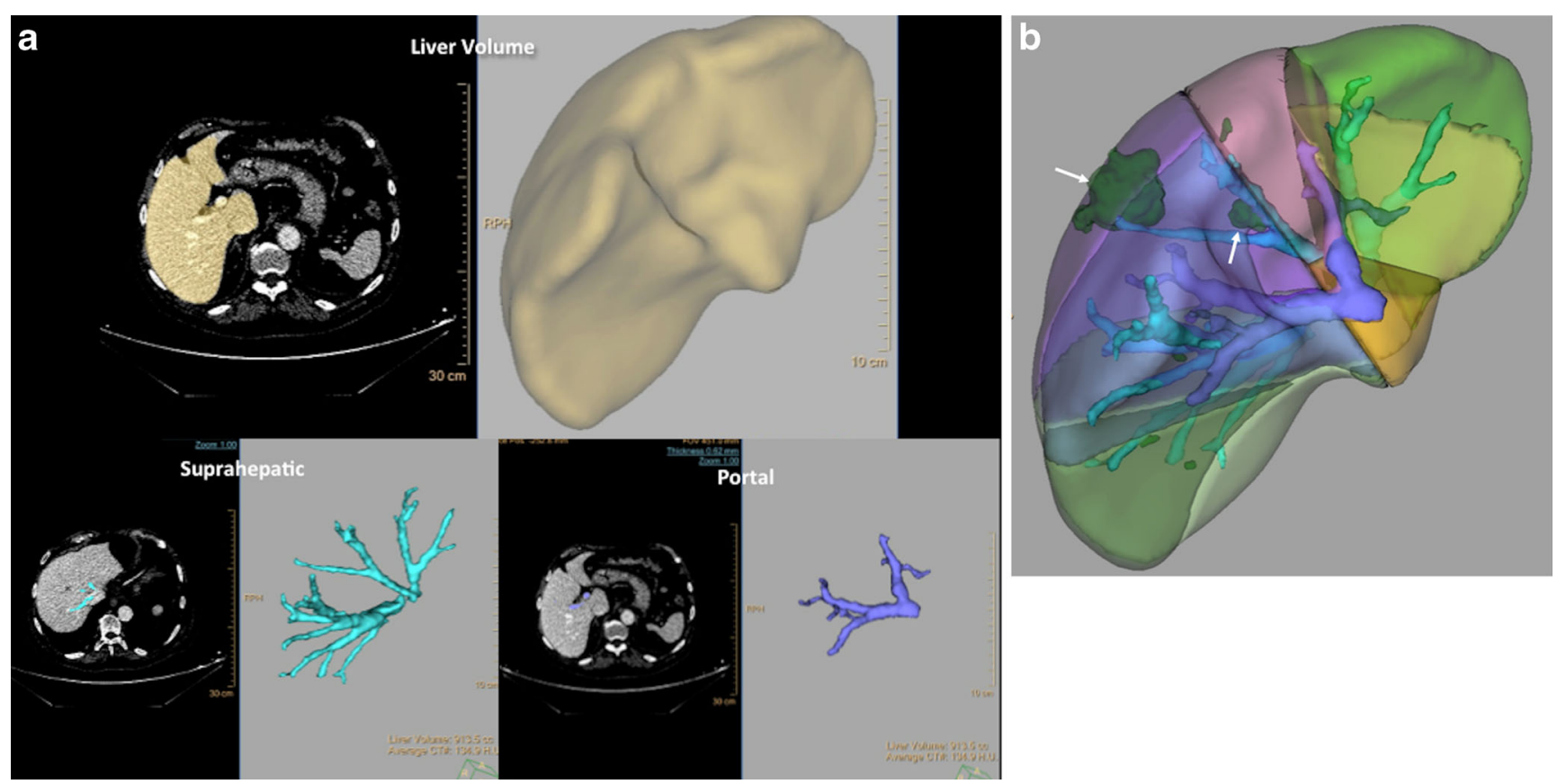

Fig. 2 CT volumetry in a patient with colorectal cancer liver metastases. Liver area determined with automated method (yellow) (top-left). Total liver volumetry $(1185 \mathrm{cc})$ (top) and images of the segmentation of the

suprahepatic and portal vessels (a). Automatic Couinaud liver segmentation using CT images (b), which allows the location of the metastatic deposits (green - white arrows)

images may be more accurate than conventional T2-weighted images to distinguish between complete and non-complete responders (sensitivity, specificity, accuracy, and area under the curve (AUC) for DCE, DWI, and T2-weighted images, respectively, 86/64/86 \%, 73/94/93\%, 79/76/93\%, and 0.76/ $0.81 / 0.90$ [19]. However, these data need to be considered with caution. Contrast uptake can be prominent in areas with inflammation, altering DCE-based measurements, and susceptibility artifacts and bright areas on high $b$-values images due to

the T2 shine-through effect can make the tumour segmentation on DWI not accurate.

Hepatic resection has improved the survival of patients with metastatic CRC. Approximately $25 \%$ of newly diagnosed patients with CRC have liver metastases at the time of diagnosis and another $25 \%$ will develop liver metastases during the course of the disease. There are several key features to consider when planning hepatic resection, including the number of segments involved,

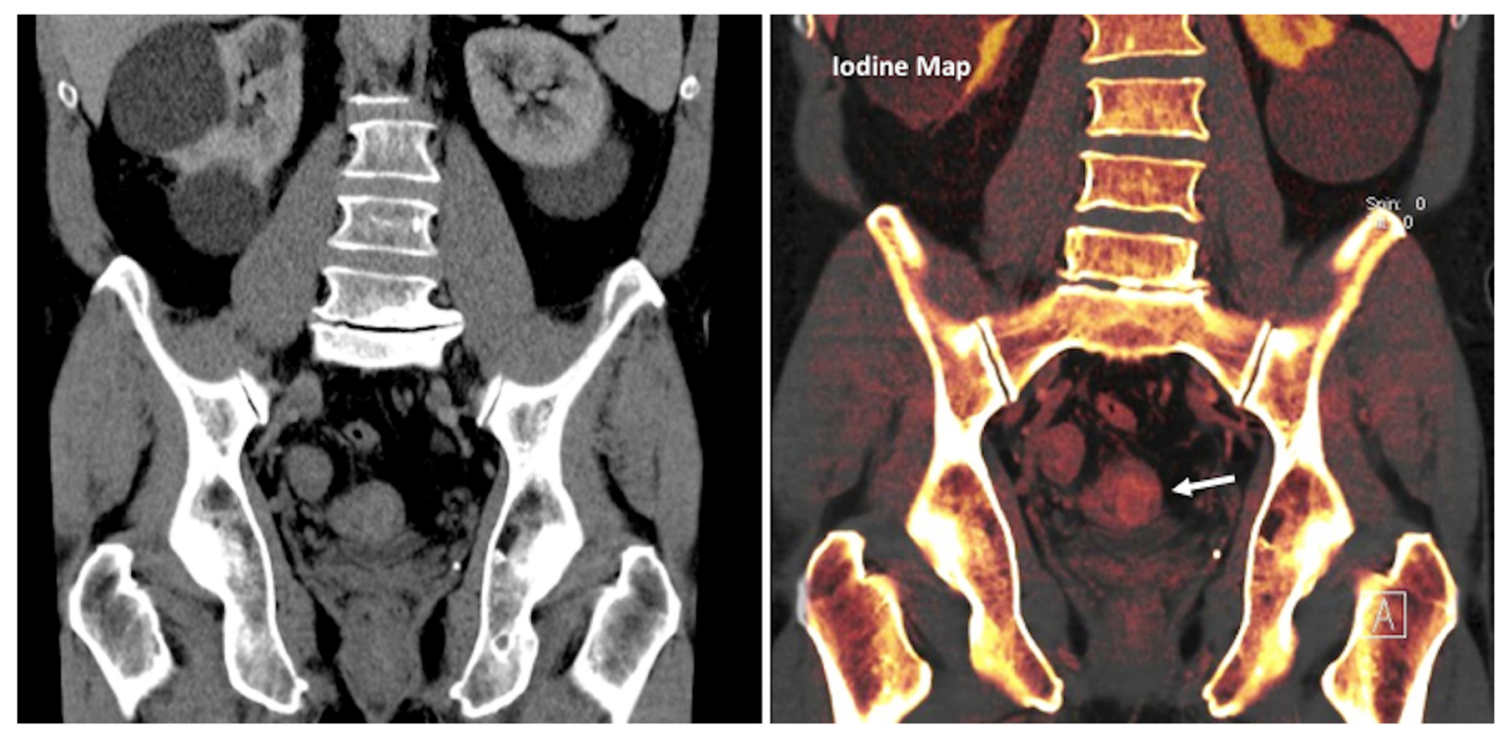

Fig. 3 Dual-energy CT in a patient with a rectosigmoid tumour. Coronal reformatted conventional CT (left) and iodine map images. Iodine map depicted iodine uptake in a mass in the sigmoid colon (arrow), improving tumour detection on CT exams without bowel preparation 


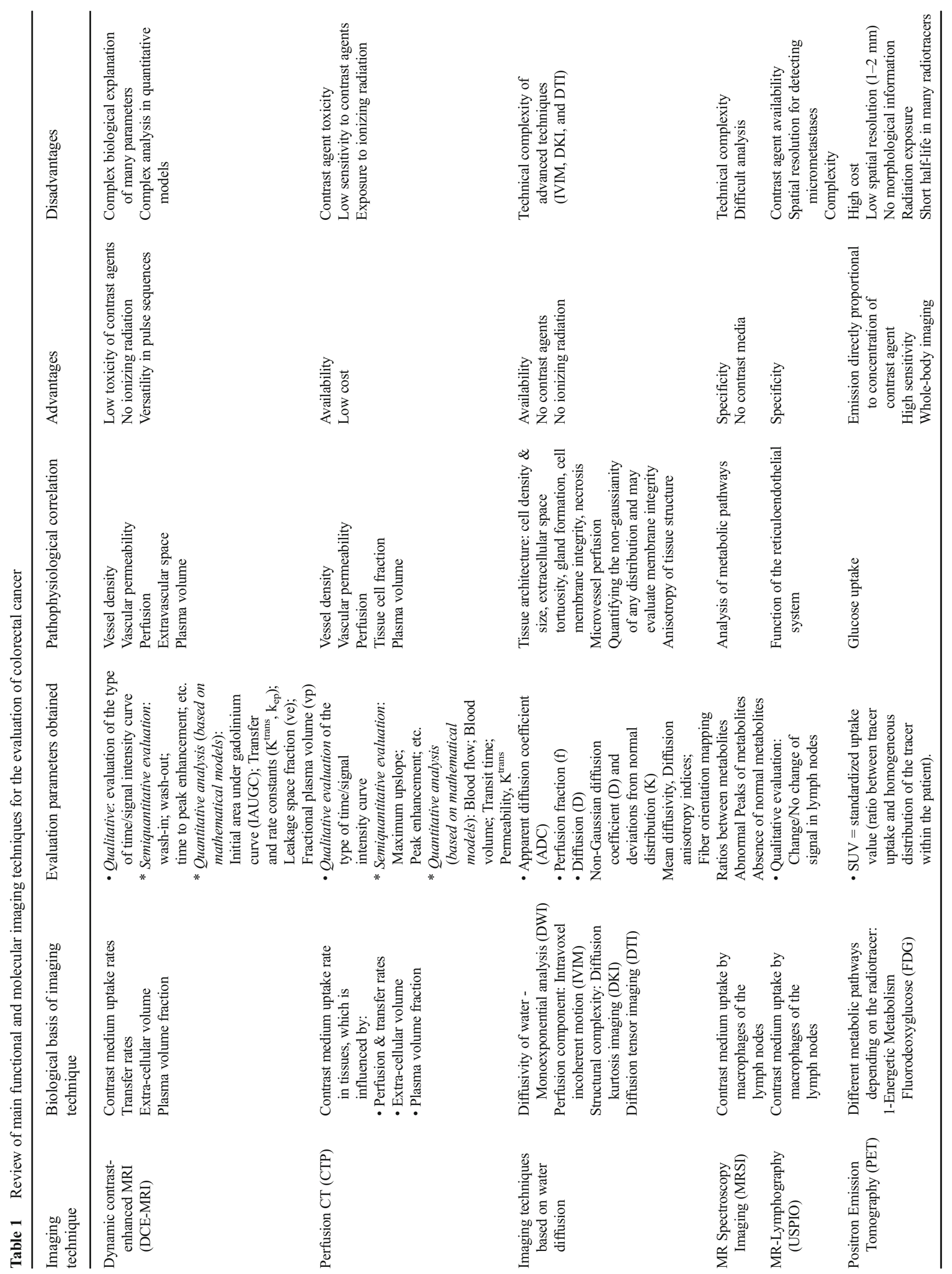




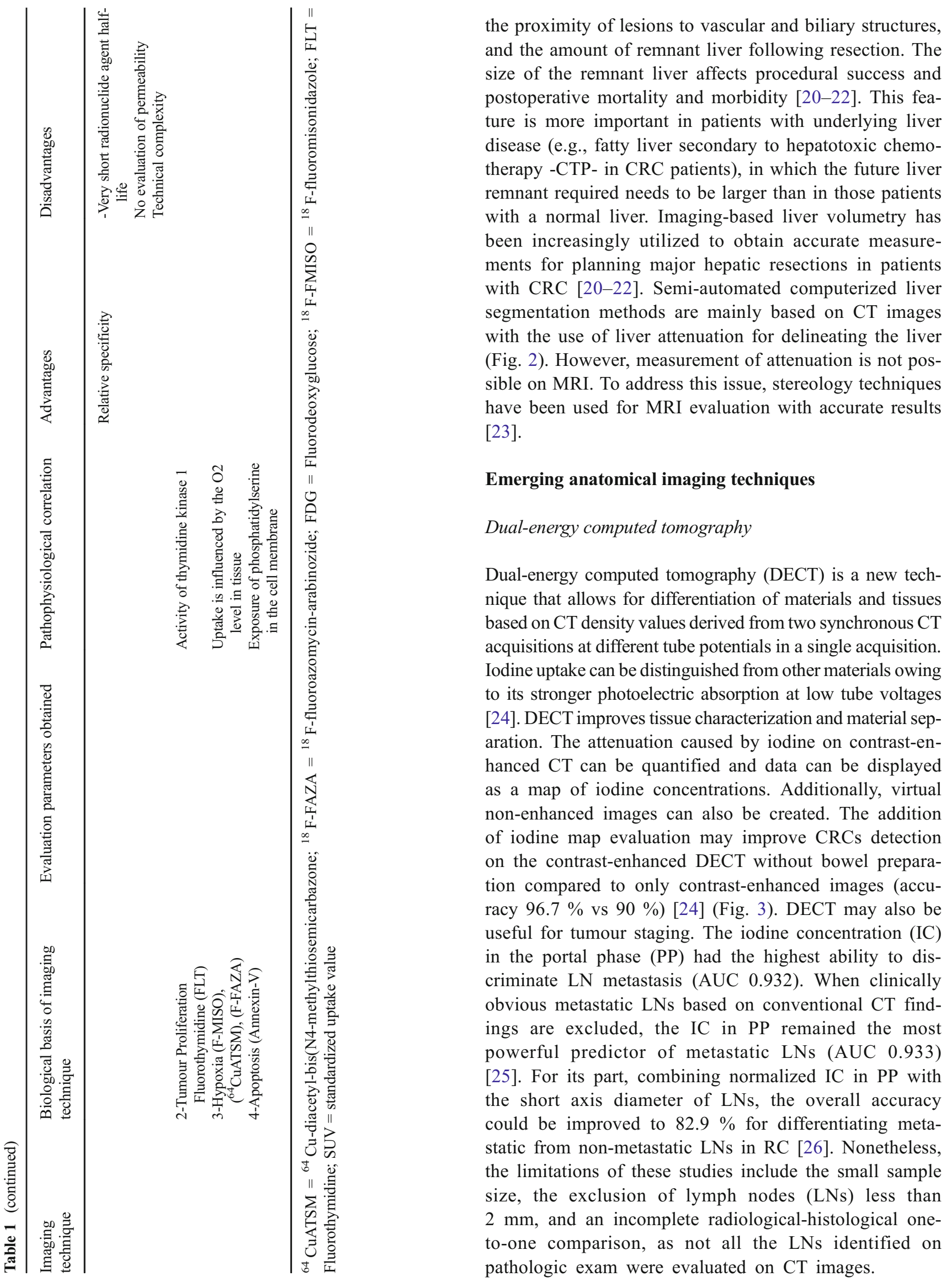


Fig. 4 Dynamic contrastenhanced (DCE) MRI evaluation of a rectal malignant tumour (white arrow) with different types of parameters. Data analysis of DCE-MRI data may be based on different approaches. A qualitative evaluation is based on the visual assessment of tumour enhancement (top left) or type of curve enhancement (top right). Semiquantitative parameters can characterize the shape and structure of the curves of enhancement (middle row). Finally, a quantitative approach is able to measure physiological parameters (bottom)
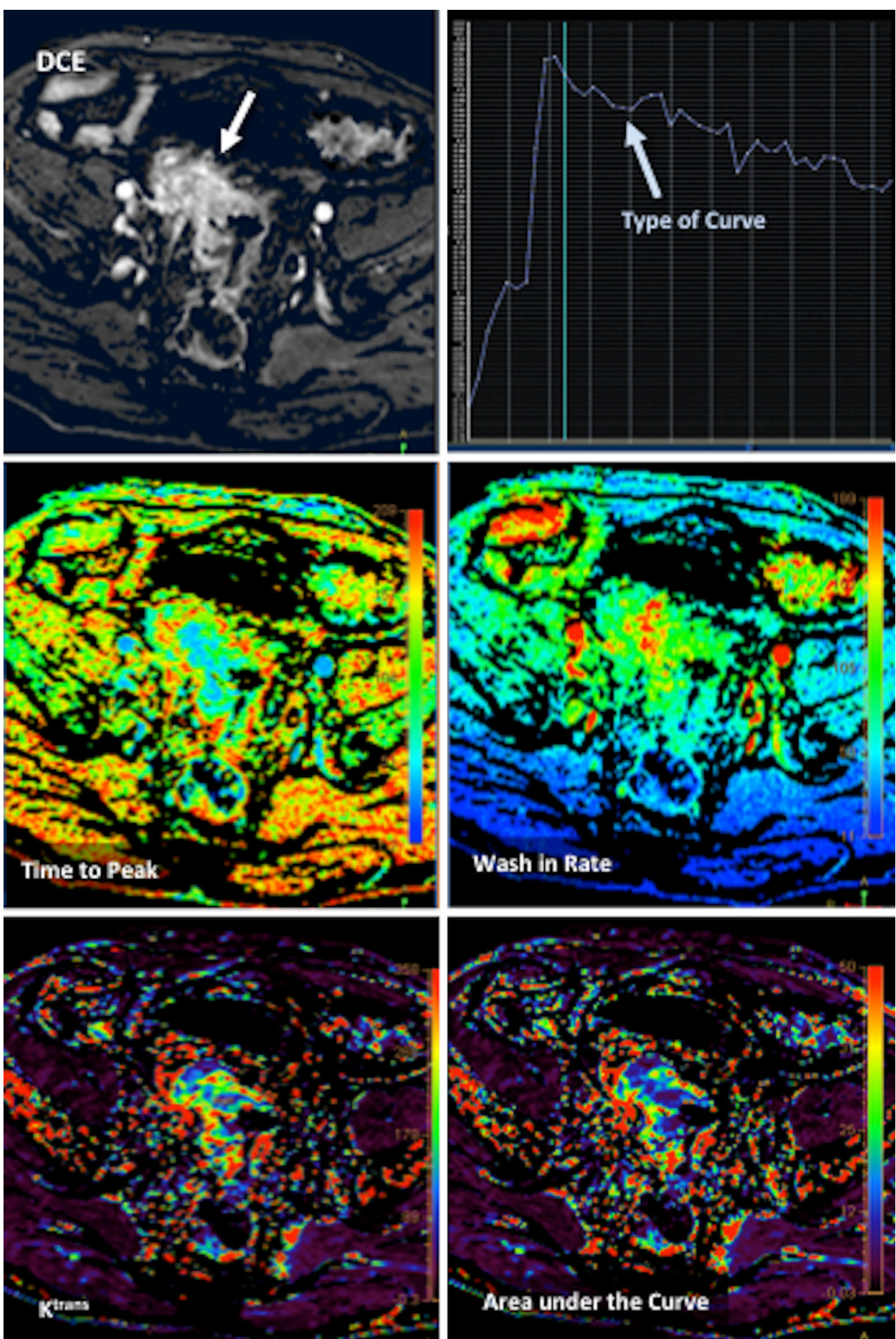

\section{Texture analysis}

Malignant tumours display a great spatial and temporal heterogeneity in their biological characteristics and behavior. However, much of the heterogeneity visible on imaging may represent noise. Texture analysis can reduce the effect of noise in images, while enhancing biologic heterogeneity. Texture analysis focuses on the distribution and relationships of grey-level values within images. Texture extracts basic components (i.e., spatial, frequency, etc.) from conventional images, creates a derived set of sub-images, and allows the quantification of different parameters, including entropy, kurtosis, and standard deviation of the pixel distribution histogram [27]. In the case of $\mathrm{CRC}$, texture features have been shown 


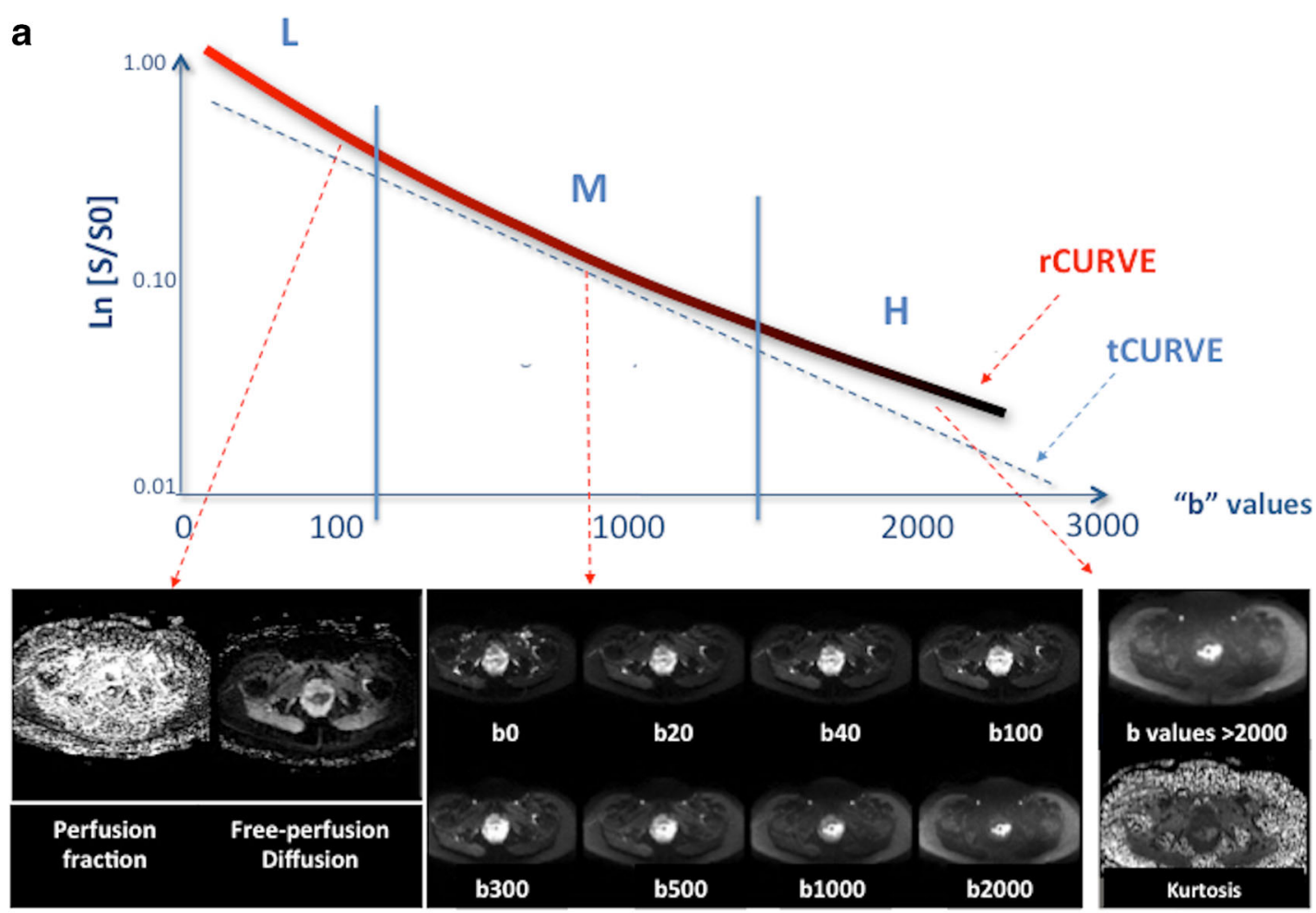

b

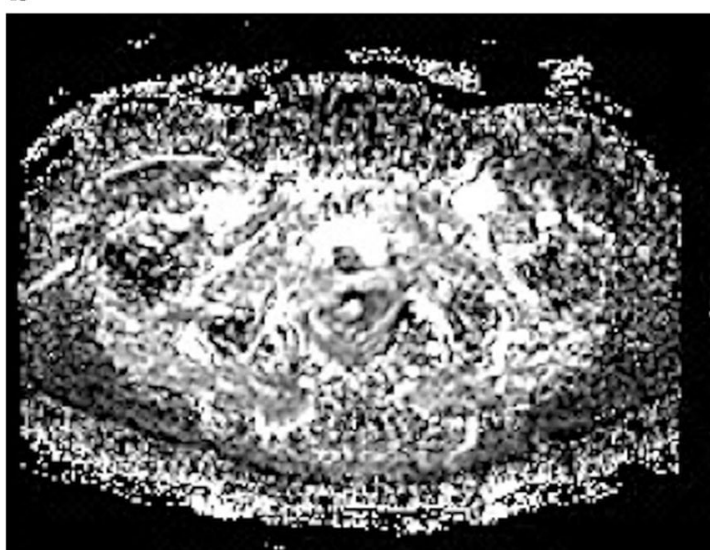

Perfusion fraction

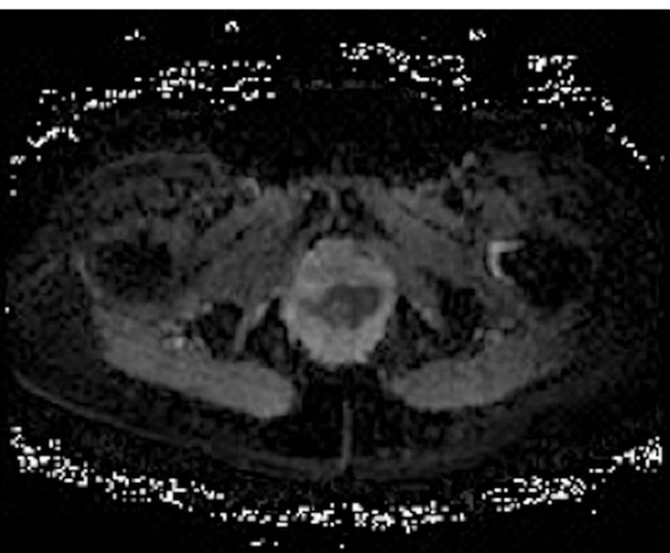

Free-perfusion diffusion

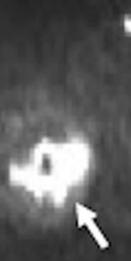

High b values ( $>2000 \mathrm{~s} / \mathrm{mm}^{2}$ )

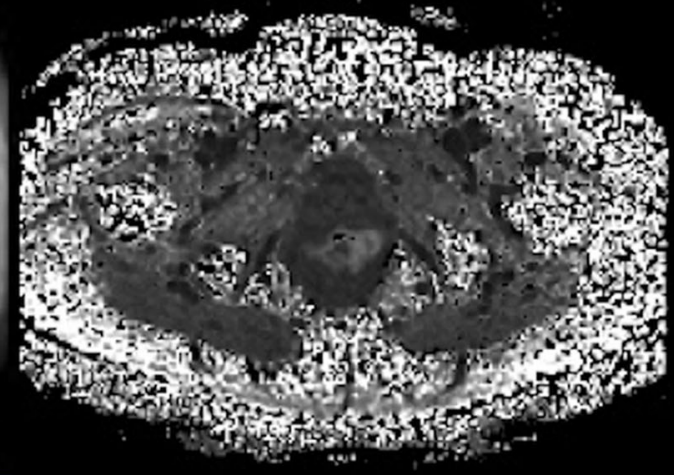

Kurtosis 
4 Fig. 5 Diffusion analysis in a rectal tumour. Analysis of the relationship between signal attenuation in tissues with different b-values. Theorical (tCURVE) and real (rCURVE) curves of signal decay are different. (a) At low b-values (L), the signal is suppressed by small diffusion weightings (e.g., b value $\leq 100 \mathrm{~s} / \mathrm{mm}^{2}$ ), which can be attributed to microcapillary perfusion, and intravoxel incoherent motion (IVIM) analysis may quantify the diffusion and perfusion effects separately. At medium b values (M) (100-1000 s/mm²), signal decay usually shows a Gaussian diffusion behavior, which would result in linear decay of the natural logarithm of the DWI signal intensity (SI) as the b-value increases, and subsequent quantification can be performed using a mono exponential analysis. On its part, at high b-values (e.g., $>2000 \mathrm{~s} / \mathrm{mm}^{2}$ ) (H), diffusion restriction is mainly secondary to cellularity, and quantification of nongaussianity for water diffusion is possible based on diffusional kurtosis imaging (DKI), which may evaluate tissue structure that creates diffusion barriers and compartments. Tumours usually show an increased kurtosis. Pictured is an example of bi-exponential analysis of diffusion in a rectal tumour (arrow) (b), which allows the calculation of perfusion-related parameters, including the perfusion fraction (f) and perfusion-free diffusion (D) (top row), and an image of tumour kurtosis (bottom right) to correlate with KRAS expression, patient's survival, tumour staging, and tumour response [28-30]. Cui et al. reported that CT texture evaluation of LNs in CRC patients demonstrated a greater heterogeneity (the fraction of pixels that deviate more than a certain range, $10 \%$ default, from the average intensity) in malignant nodes with a size greater than $3 \mathrm{~mm}$ and less than $10 \mathrm{~mm}$ and fractal dimension [28]. However, in contrast, lessheterogeneous tumours have been associated with a poorer outcome. In this sense, $\mathrm{Ng}$ et al. reported that primary CRC at fine filter levels in contrast-enhanced CT were showed a poorer 5-year overall survival (OS) rate with values for entropy, kurtosis, and standard deviation of pixels of less than 7.89, 2.48 , and 61.83 , respectively, and at least 0.01 for uniformity and -0.38 for skewness [29]. Luebner et al. demonstrated that CRC tumours with hepatic metastases that are more homogeneous at coarse filters (less entropy, smaller standard deviation, higher in attenuation/higher mean of positive pixels) are potentially more aggressive in their biology with higher tumour grade and poorer OS [30]. To explain this apparent

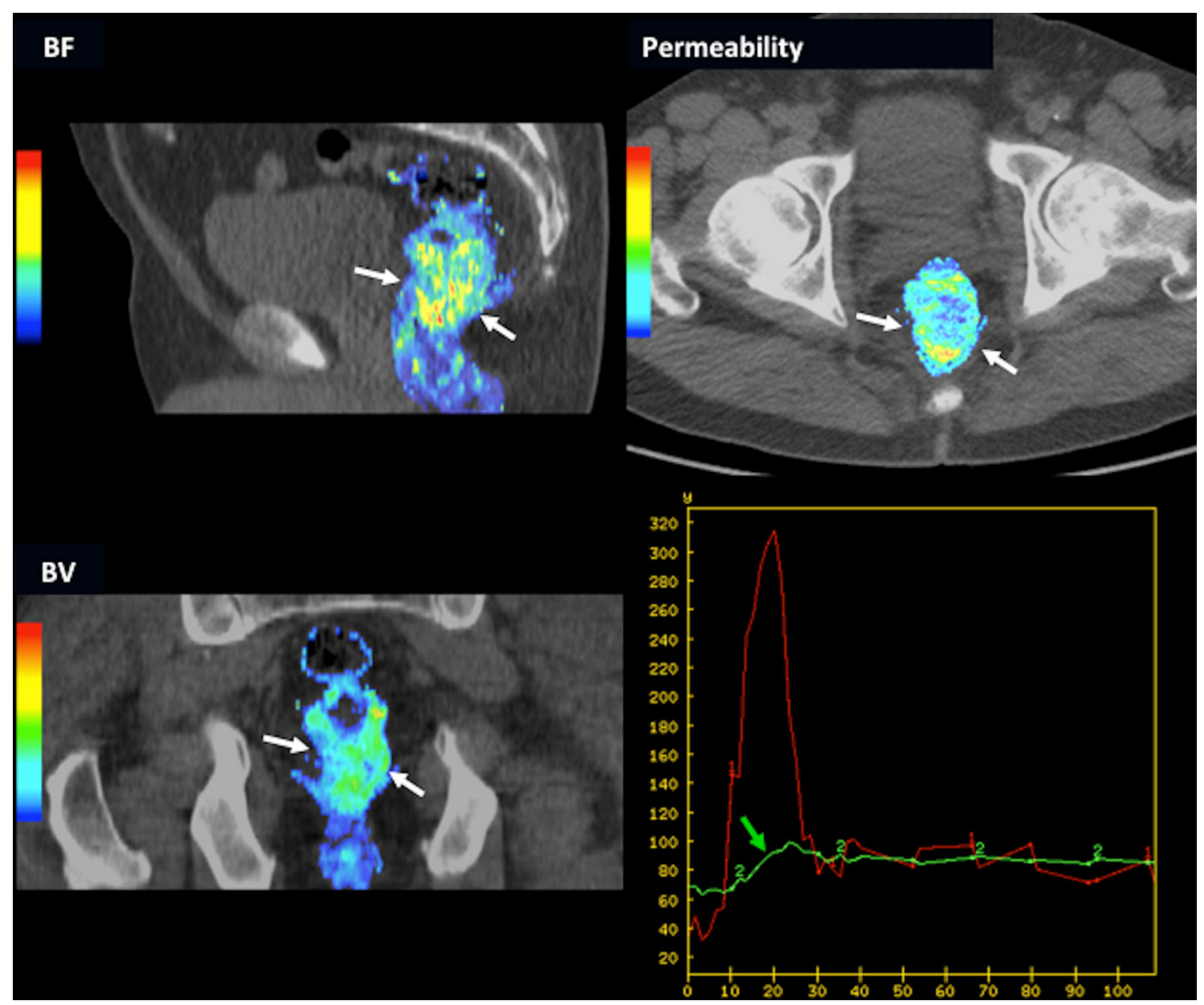

Fig. 6 Volumetric perfusion CT multiplanar and multiparametric evaluation in a 62-year-old man with rectal cancer (white arrows). Parametric maps fused (50\% transparency) with $\mathrm{CT}$ images in different planes demonstrated increased values of perfusion-related parameters within the tumour: sagittal reformatted blood flow (BF) map, axial reformatted permeability map, and coronal reformatted blood volume (BV) map. Time-density curve of the tumour (bottom-right) demonstrated an enhancement curve type 3 (green arrow) 
Table 2 Biological correlation of perfusion-related parameters

\begin{tabular}{|c|c|c|}
\hline BV and BF (often coupled) & Permeability & Biological interpretation \\
\hline$\uparrow \uparrow \uparrow$ & $\downarrow$ or $\leftrightarrow$ or $\uparrow$ & $\begin{array}{l}\text { New vessel formation (increased perfusion and blood volume) } \\
\text { with varying degrees of maturation }\end{array}$ \\
\hline$\downarrow$ or $\leftrightarrow$ or $\uparrow$ & $\uparrow \uparrow \uparrow$ & $\begin{array}{l}\text { Poor perfusion with increased permeability (immature vessels), } \\
\text { which usually represent a reaction to hypoxia }\end{array}$ \\
\hline$\uparrow$ & $\leftrightarrow$ or $\downarrow$ & Mature vasculature \\
\hline$\uparrow$ & $\leftrightarrow$ or $\uparrow$ & Inflammation $+/-$ early fibrosis \\
\hline$\downarrow$ & $\downarrow$ & $\begin{array}{l}\text { Mature fibrotic areas (usually showing a progressive } \\
\text { enhancement) }\end{array}$ \\
\hline$\downarrow \downarrow$ & $\downarrow \downarrow$ & Poor vascularized areas \\
\hline $\mathrm{BF} \downarrow$ relating to $\mathrm{BV}$ & - & $\begin{array}{l}\text { Blood flow-blood volume mismatch, which usually } \\
\text { represents hypoxia }\end{array}$ \\
\hline$+/-0$ & $+/-0$ & Necrotic areas \\
\hline
\end{tabular}

Note - Data from literature reviews and personal experience and adapted from references 30,31, and 45 $\mathrm{BV}=$ blood volume, $\mathrm{BF}=$ blood flow contradiction between studies, we must consider the limitations of texture analysis, which include that biologic correlates of texture analysis have not been definitively confirmed in histological studies and that image acquisition parameters and analysis (unenhanced vs contrast-enhanced exam, pattern of contrast administration, texture methods, software platforms, etc.) deeply affect measurement of texture features and change their biological correlation (i.e., texture features may reflect cellular distribution on unenhanced images at a fine scale; they may also reflect the distribution of the contrast agent between the intra- and extravascular extracellular space (EES) on contrast-enhanced images) [29].

\section{Functional and molecular imaging for the evaluation of tumour hallmarks in CRC}

CRC typically show characteristic tumour phenotypic alterations, which are manifestations of genetic changes and metabolic reprogramming, including sustained angiogenesis, limitless replication potential, and altered metabolic pathways (including an increased glycolytic capacity) [5]. Anatomic imaging techniques may be insensitive to mapping the distribution of these tumour-specific characteristics. FMI-derived techniques may help to discriminate these features for clinical decision-making $[6,8]$ (Table 1).

\section{Imaging of tumour angiogenesis in CRC}

Angiogenesis is a prognostic feature in CRC that has been correlated with important tumour characteristics such as grade and stage and with an increased incidence of metastases and local tumour recurrence. Functional imaging techniques may provide additional insights into the tumour microenvironment. Main imaging techniques for assessing tumour vascularization in the clinical field are DCE techniques based on MRI and CT. These techniques acquire a series of images through a region of interest before, during, and after the intravenous injection of a contrast

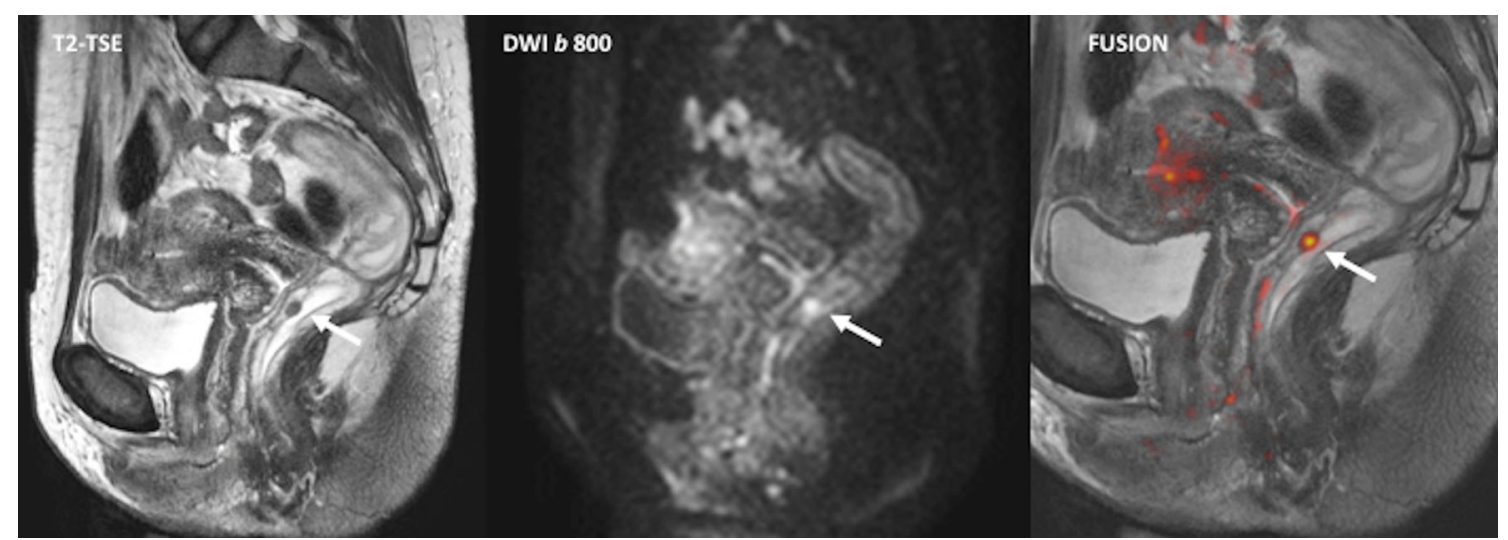

Fig. 7 Diffusion for detecting colorectal tumours. A carcinoid tumour (white arrows) in a 44-year-old patient. Sagittal fast spin-echo T2weighted image (left) and sagittal diffusion-weighted image with high $\mathrm{b}$ value $\left(b=800 \mathrm{~s} / \mathrm{mm}^{2}\right)$ (center) showed a rectal tumour nodule. Fused image (right) superimposing sagittal T2-weighted MR image and colorcoded map derived from high-b-value diffusion-weighted image clearly delineated the tumour 
media [31, 32]. Different method of analysis can be used to evaluate the obtained data, from purely qualitative assessment to complex mathematical modeling. However, not all the studies have shown agreement concerning the capability of imaging to reflect angiogenesis in CRC [33]. Published data related to the correlation of DCE-derived perfusion parameters with morphologic, angiogenic, and molecular prognostic factors in RC results are sometimes contradictory [8, 34-37]. However, DCE techniques may show clinical value in diagnosis, prognosis, planning therapy, assessment of response to treatment, and detection of tumour relapse in CRC patients $[6-8,34,35]$.

\section{MR-based imaging techniques}

T1-weighted DCE-MRI techniques are able to evaluate tissue perfusion and vascular leakage based on signal changes secondary to the presence of low-molecular-weight contrast media in the EES [6-8, 31] (Fig. 4). Different parameters can be obtained depending on the complexity of the analytic model applied. Quantitative parameters such as the transfer constant $\left(\mathrm{K}^{\text {trans }}\right)$ have demonstrated a prognostic value. Lim et al. reported that $\mathrm{K}^{\text {trans }}$ values in rectal tumours at presentation in the downstaged group following CRTP were significantly higher than those in
Fig. 8 Whole-body diffusion imaging for tumour staging. Conventional portal-phase contrast-enhanced images (a) demonstrated two liver metastases (left - red arrows) and a tumour in the cecum (right white arrow). Whole-bodydiffusion-weighted image with inverted gray scale (b) depicted both focal liver lesions (red arrows) and the tumour in the cecum (white arrow) that showed restriction of diffusion. ${ }^{18}$ F-FDGPET image (c) evidenced similar findings
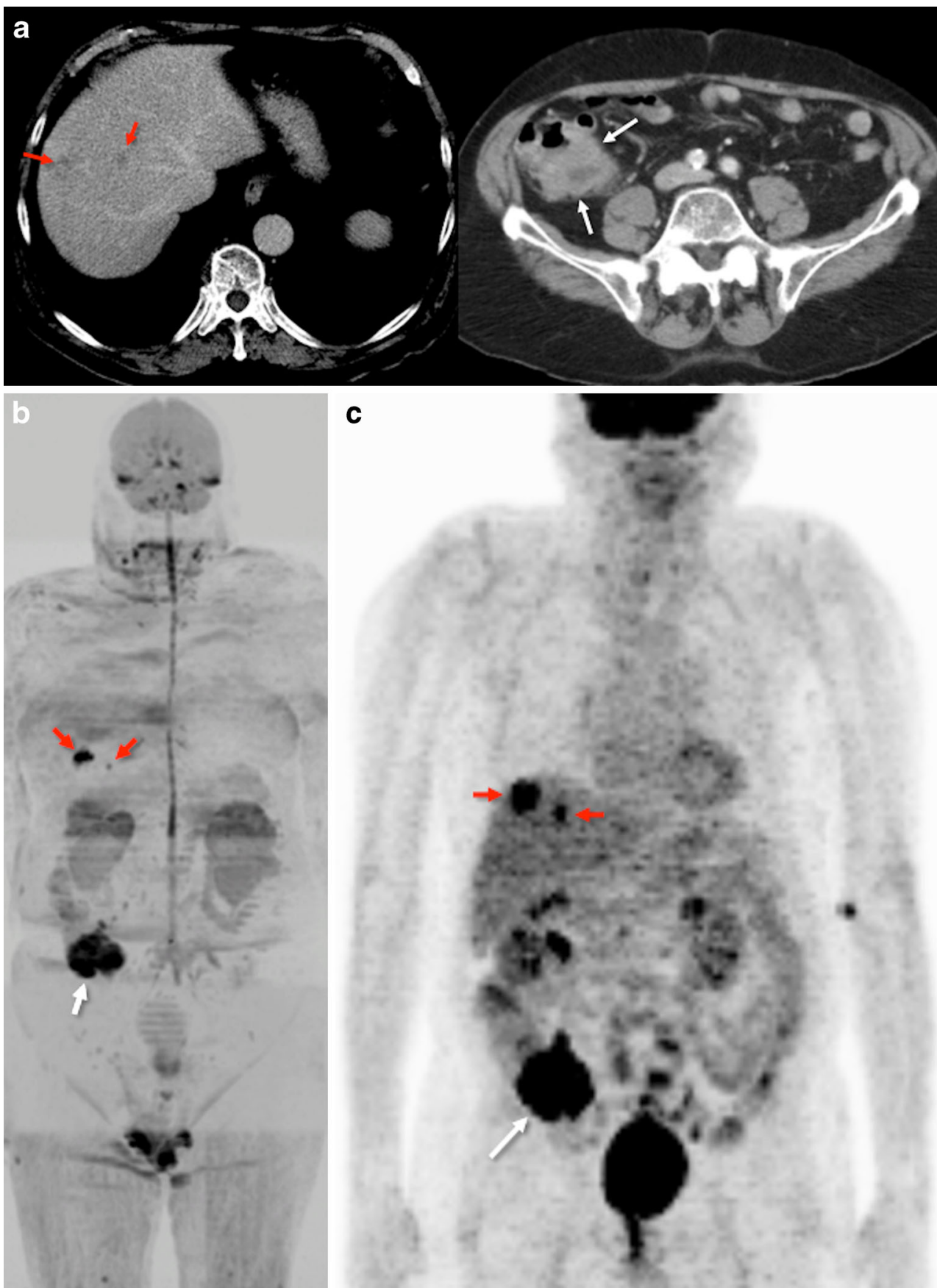

C 
the non-downstaged group [38]. In contrast, Gollub et al. in a similar study did not find that pretreatment $\mathrm{K}^{\text {trans }}$ had predictive value, though post-treatment $\mathrm{K}^{\text {trans }}$ in a select group was able to distinguish pathological complete responders from incomplete responders [39]. However, different therapies were used in both studies, which could explain these differences. CRTP was used in Lim's study while CTP/antiangiogenic therapy (bevacizumab) was administrated in Gollub's study (which may cause a more effective devascularization of the tumour, resulting in lower $\mathrm{K}^{\text {trans }}$ values). DCE-MRI with the use of blood pool contrast-agents has shown a good performance in $\mathrm{RC}$ prognosis of tumour response to CRTP. The late slope was able to discriminate between good and poor responders with an AUC of 0.90 , sensitivity of $92 \%$, specificity of $82 \%$, positive predictive value (PPV) of $80 \%$, and negative predictive value (NPV) of $93 \%$ [40]. Evaluation of tumour pathological response after CRTP is another important role of imaging. In this setting, the relative change in $\mathrm{K}^{\text {trans }}$ has shown a good predictive potential using a cutoff value of $32 \%$ reduction in median $\mathrm{K}^{\text {trans }}$ with a PPV of $100 \%$ for good response (complete pathologic response -pCR- and near-pCR based on the Mandard's tumour regression grade) [41], and Tong et al. reported that a $\mathrm{K}^{\text {trans }}$ threshold of 0.66 could distinguish between complete and incomplete response before CRTP in RC with a sensitivity of
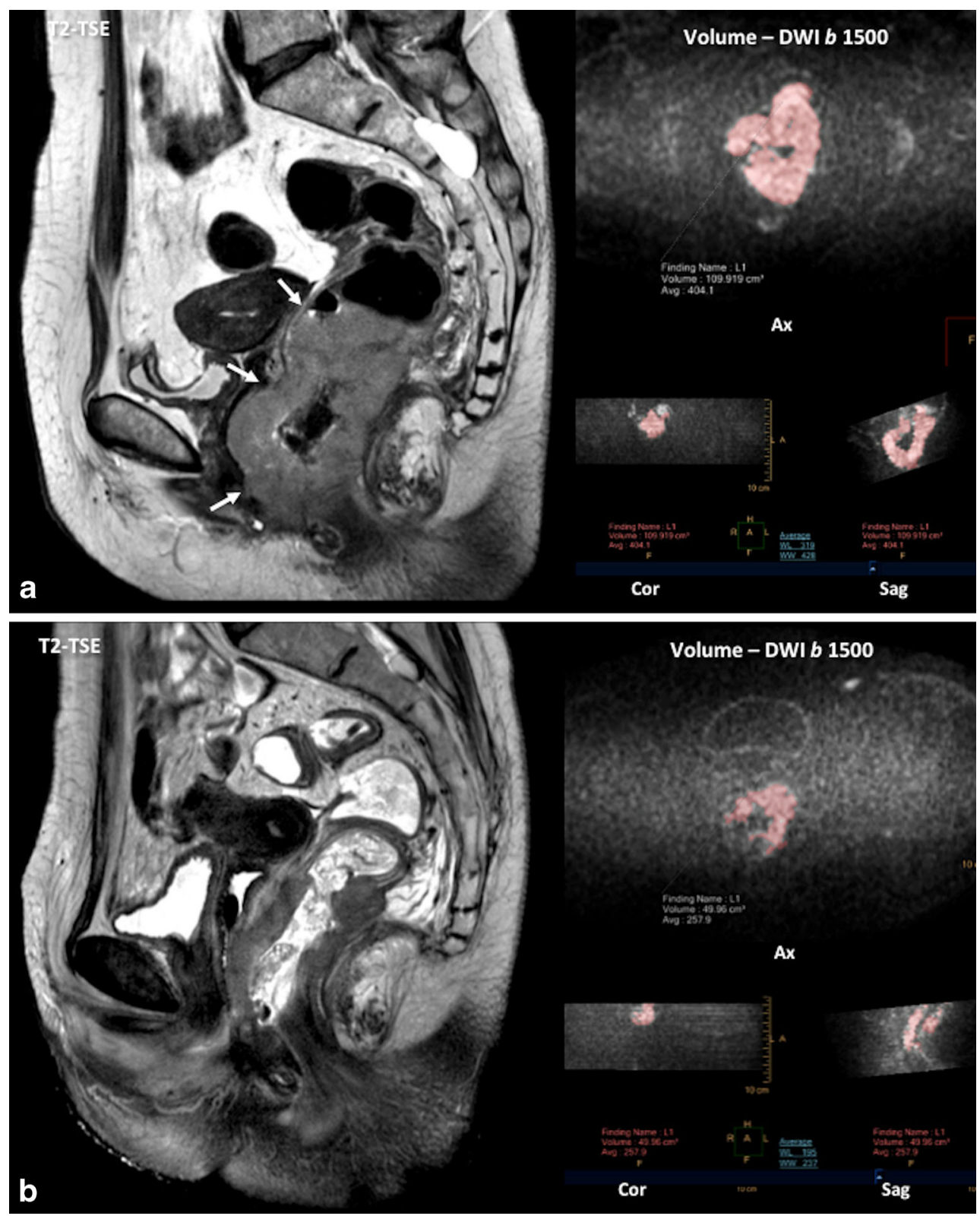

Fig. 9 Diffusion for the evaluation of tumour response. Pretherapy (a) sagittal T2-TSE image and volumetry (volume $=109.91 \mathrm{cc}$ ) based on diffusion $\left(b=1500 \mathrm{~s} / \mathrm{mm}^{2}\right)$ image showed a big rectal cancer with invasion of the anal canal (white arrows). Post-therapy (b) volume was reduced $($ volume $=42.96 \mathrm{cc}$ ). Histogram analysis $\odot$ pre and post-therapy of the apparent diffusion coefficient (ADC) also demonstrated increased $\mathrm{ADC}$ values 
$100 \%$ [42]. Finally, DCE-MRI findings correlate with clinical outcome in patients who undergo surgical treatment for recurrent RC. A higher value correlated directly with a complete tumour-free resection margin, while in the case of the rate constant or $\mathrm{k}_{\mathrm{ep}}$ there was a negative correlation [43]. However, it must be considered that clinical comparability across perfusion analysis solutions is currently not warranted. A considerable variability for DCE-MRI pharmacokinetic parameters has been found among various commercially available perfusion analysis solutions [44].

DWI can be also used for assessing tumour vascularization. The perfusion component dominates the signal decay at low bvalues and can be assessed using intravoxel incoherent motion (IVIM) analysis (Fig. 5). This feature enables differentiation between the perfusion fraction (f) and perfusion-free diffusion (D). The IVIM-related parameters may be used in the noninvasive evaluation of tumour perfusion. Bäuerle et al. found that $\mathrm{f}$ correlated to the vascular area fraction (percentage of CD 31 positive-staining area) on histological evaluation in the normal rectum and in tumours [45]. However, this correlation or significant changes on $\mathrm{f}$ have not been evidenced after CRTP $[45,46]$, which restricts the clinical value of IVIM in CRC.

\section{Perfusion CT}

DCE-CT or perfusion CT (PCT) is an attractive technique for the evaluation of tumour vasculature based on the temporal change in tumour enhancement following intravenous iodinated contrast agent administration. Pharmacokinetic models allow obtaining qualitative data and quantitative parameters on tumour vascularization including blood flow (BF), blood volume (BV), mean transit time (MTT), permeability-surface area, or $\mathrm{K}^{\text {trans }}[34,35]$ (Fig. 6). Tumour perfusion-related parameters (BF) may also distinguish the normal colonic wall from CRC $(10-40 \mathrm{ml} /$ $\mathrm{min}^{-1} 100 \mathrm{~g}^{-1}$ tissue vs. $50-200 \mathrm{ml} / \mathrm{min}^{-1} 100 \mathrm{~g}^{-1}$ tissue, respectively) [34]. Variations in tumour phenotyping and intratumoural heterogeneity may also be assessed based on the combination of perfusion-related parameters (Table 2) [34, 47], although the use of global mean values for perfusion parameters may underestimate the extent of spatial heterogeneity. In clinical practice, CT perfusion-related parameters may separate well, moderately, and poorly differentiated RC. Sun et al. reported that the mean BF was significantly different among well, moderately, and poorly differentiated groups $(61.17 \pm 17.97,34.80 \pm 13.06$, and 22.24 $\pm 9.31 \mathrm{~mL} /$ minute $/ 100 \mathrm{~g}$, respectively) [48]; while Kim et al.

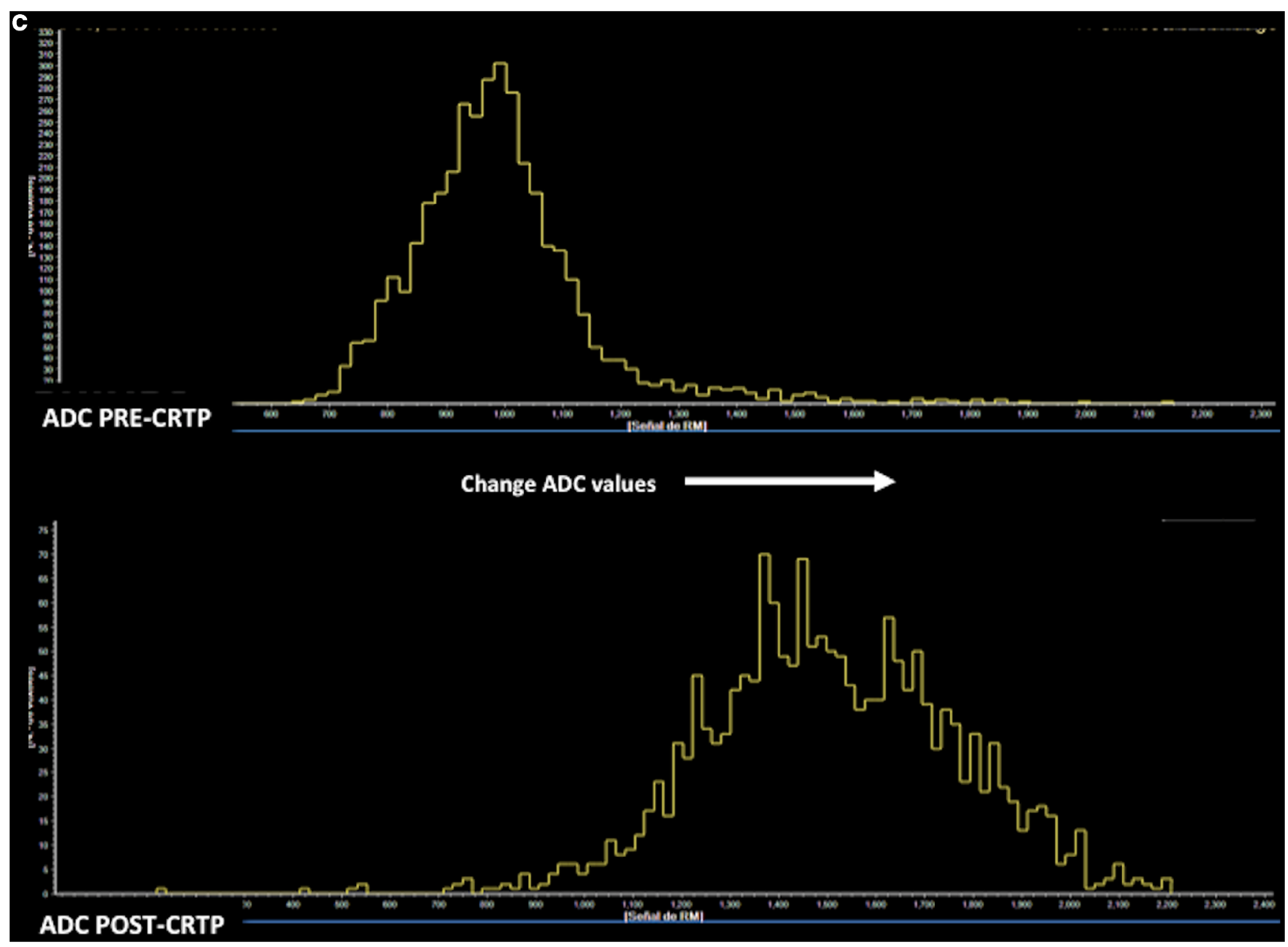

Fig. 9 (continued) 
evidenced that BF was higher in moderately differentiated CRC than well differentiated and poorly differentiated CRC [49] Although, certain limitations of these studies must be considered. First, the study sample size was small and second, there was no precise correlation between tumour ROI in perfusion CT and pathologic specimen after surgery. PCT parameters may also have a role as a potential prognostic biomarker in CRC. Hayano et al. evidenced that patients with more poorly perfused RCs ( $<40 \mathrm{ml} / \mathrm{min}^{-1} 100 \mathrm{~g}^{-1}$ tissue) had a poorer outcome [50]. Finally, PCT can also depict therapy-induced modifications in the vascularization of CRC [34, 35, 47, 51]. Changes in tumour vascularization depend on both the therapeutic mechanism of action and the timing of response evaluation. In this setting, neoadjuvant CRTP produces a decrease in perfusion parameters (reduction in BF around $40 \%$ ), although an early temporal increase can be depicted secondary to radiation-induced inflammatory changes [52]. In the case of CRC liver metastases treated with therapeutic regimens including antiangiogenic agents, several studies also have shown a reduction in BF and permeability after the therapy $[53,54]$.

PCT is actually a robust technique on the basis of recent technological advances, including whole-tumour coverage, motion correction, noise reduction algorithms, etc. However, quality control is essential to enable CT quantification and efforts are needed toward a standardization of acquisition and data processing.

Finally, many features have to be considered that can affect the calculation of vascular parameters in both CT and MRI, including the conditions of signal production, the characteristics of the contrast agent, and the data analysis model. There are several important differences between DCE-MRI and PCT. The attenuation (expressed in Hounsfield units) is directly proportional to contrast agent concentration on CT. For its part, on MRI, the image intensities depend on many features (underlying native signal of the tissue or various parameters of the imaging sequences) adding complexity to analysis. Beside this, physiological explanation of some MRI-based quantitative parameters is complex, while PCT usually provides a series of more comprehensive parameters. CT offers a wider availability, but also shows potential limitations, including radiation dose, contraindications to iodinated contrast media, or the fixed axial plane of CT scanning. In the case of DCEMRI, this technique is recommended over PCT for relatively young patients and offers a better signal-to-noise ratio, a stronger contrast uptake, and the absence of ionizing radiation $[6-8,31]$.

\section{Imaging tumour proliferation and cellularity in CRC}

A basic characteristic of cancer is uncontrolled cell proliferation, which generally causes a greater cell density in tumour lesions.
Functional imaging of cellularity: Diffusion-weighted imaging in $C R C$

To date, DWI is a basic technique in oncologic imaging [55]. Diffusion measures the random Brownian motion of water molecules within a voxel of tissue. The relationship between histology and diffusion is complex. DWI can mainly provide an indirect evaluation of cellularity and the integrity of cell membranes, but gland formation, perfusion, or cell death may also influence water diffusion. Besides, diffusion can be quantitatively assessed using the apparent diffusion coefficient (ADC) value. In $\mathrm{CRC}$, this technique has shown to be of value for tumour detection, staging, prognosis, evaluation of response, and assessment of recurrence. DWI is a useful tool for detecting colorectal tumours. Ichikawa et al. reported a sensitivity and specificity of high-b-value DWI for detection of CRC of $91 \%$ and $100 \%$, respectively [56] (Fig. 7). However, this study had some limitations, including a small study population and the fact that it did not include other benign conditions (i.e., inflammatory bowel disease), which could reduce its specificity. DWI may also improve tumour staging. This technique increases the sensitivity for detecting LNs. In a study by Heijnen et al., DWI detected $6 \%$ more nodes than T2-weighted imaging [57]. Concerning the characterization of LNs, specificity and accuracy also increased after adding DWI to T2-weighted images, although the diagnostic accuracy of ADC for discriminating metastatic from non-metastatic LNs is only around $70 \%$,

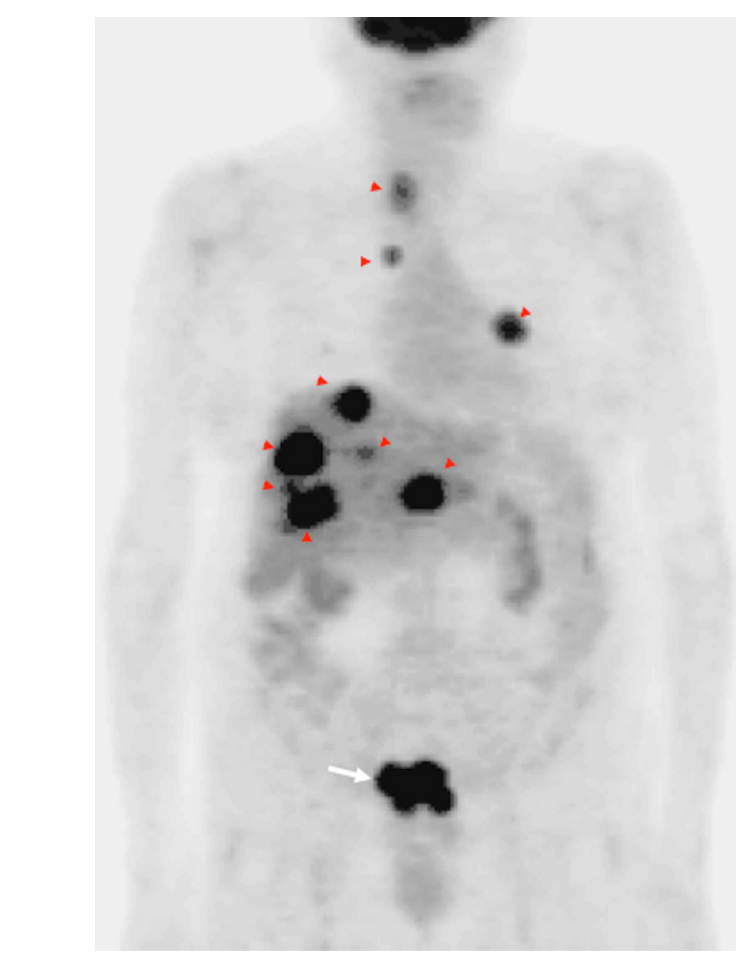

Fig. $10{ }^{18}$ F-FDG-PET in staging rectal cancer. Coronal PET image evidenced a focal area of uptake in the pelvis corresponding to the primary tumour (white arrow). PET also demonstrated multiple liver, lung, and lymph-node metastases (red arrowheads) 
because subjective visual assessment cannot discriminate between benign and malignant nodes, as both display high DWI signals with increasing b-values, and the ADC values of malignant nodes have been shown to be only slightly lower than that of benign nodes - not enough to allow their discrimination.

Besides this, complete radio-pathological correlation was not possible in published studies, and a good correlation between anatomical and DWI sequences was difficult to achieve due to partial volume effects (based on the different voxel size used in both sequences) $[58,59]$. The use of whole-body (WB)DWI may be an attractive alternative for staging CRC (Fig. 8). A small study evidenced that the overall sensitivity of WBDWI as a single modality for the detection of malignant lesions was $81 \%$. All primary CRC were detected, included $77 \%$ of the liver metastases, $72 \%$ of the distant nodal metastases, and $75 \%$ of the lung metastases [60]. Further, in a study including 28 gastrointestinal cancers (23 CRC), Gong et al. found no statistically significant difference in the overall diagnostic performances of PET-CT (accuracy $98.9 \%$; sensitivity $95.2 \%$; specificity $99.8 \%$; PPV $98.9 \%$; NPV $98.9 \%$ ) and WB-DWI (accuracy $95.9 \%$; sensitivity $81.7 \%$; specificity $99.1 \%$; PPV $95.0 \%$; NPV $96.1 \%$ ) for the initial diagnosis or post-operative follow-up in detecting distant metastases or recurrence [61].

The role of diffusion as a prognostic or predictive tool has been also evaluated. In this setting, RC with lower ADC values was associated with more aggressive tumour behavior $[62,63]$. Beside this, diffusion may predict $\mathrm{RC}$ response to neoadjuvant CRTP $[64,65]$. A significant correlation between tumour volume reduction and pre-CRTP ADC values has been reported [64]. Pre-CRTP ADC of the histopathological responders was significantly lower than that of the histopathological non-responders and the change of ADC of the responders was significantly higher. Concerning tumour response evaluation, patients with a pCR after CRTP always have a better prognosis than those with other TRG grades. Increases in ADC values occur within 3-7 days in responding patients treated with CRTP [66]. Preliminary results indicate that DWI improves the diagnostic performance of MRI to detect early tumour response and to predict mesorectal fascia tumour clearance [67]. DWI, DW-MR-volumetry, and ADC histogram analysis are significantly more accurate than T2weighted images in assessing tumour response [68-70] (Fig. 9). However, MRI showed heterogeneous results of diagnostic performances for restaging RC after CRTP, although better results were demonstrated when DWI was included [71]. A meta-analysis including 16 studies and 826 patients determined that the changes between the pre- and post-ADC are good predictors of a pCR, but some misjudgments remain, because DWI cannot reliably microscopically discriminate residual viable tumour cells from fibrosis, which can cause a considerable overlap of the ADC values between a pCR and near-pCR. Furthermore, DWI sensitivity is low, mainly due to the erroneous interpretation of high signals in 'normal' posttreatment rectal walls as residual tumour $[72,73]$. These data are in agreement with a systematic review of the role of imaging (including DWI and fluorodeoxyglucose $\left({ }^{18} \mathrm{~F}-\mathrm{FDG}\right)$
Fig. $11{ }^{18}$ F-FDG-PET for evaluating response to therapy. Changes secondary to therapy in a patient with rectal cancer treated with chemoradiation. Sagittal ${ }^{18} \mathrm{~F}$ FDG-PET-CT image pretherapy (left) demonstrated an FDG-avid rectal mass (white arrow). Postneoadjuvant chemoradiotherapy ${ }^{18}$ F-FDG-PET-CT image evidenced a complete tumour response (Courtesy JM LlamasElvira, MD. Department of Nuclear Medicine, Hospital Universitario Virgen de las Nieves, Granada, Spain)
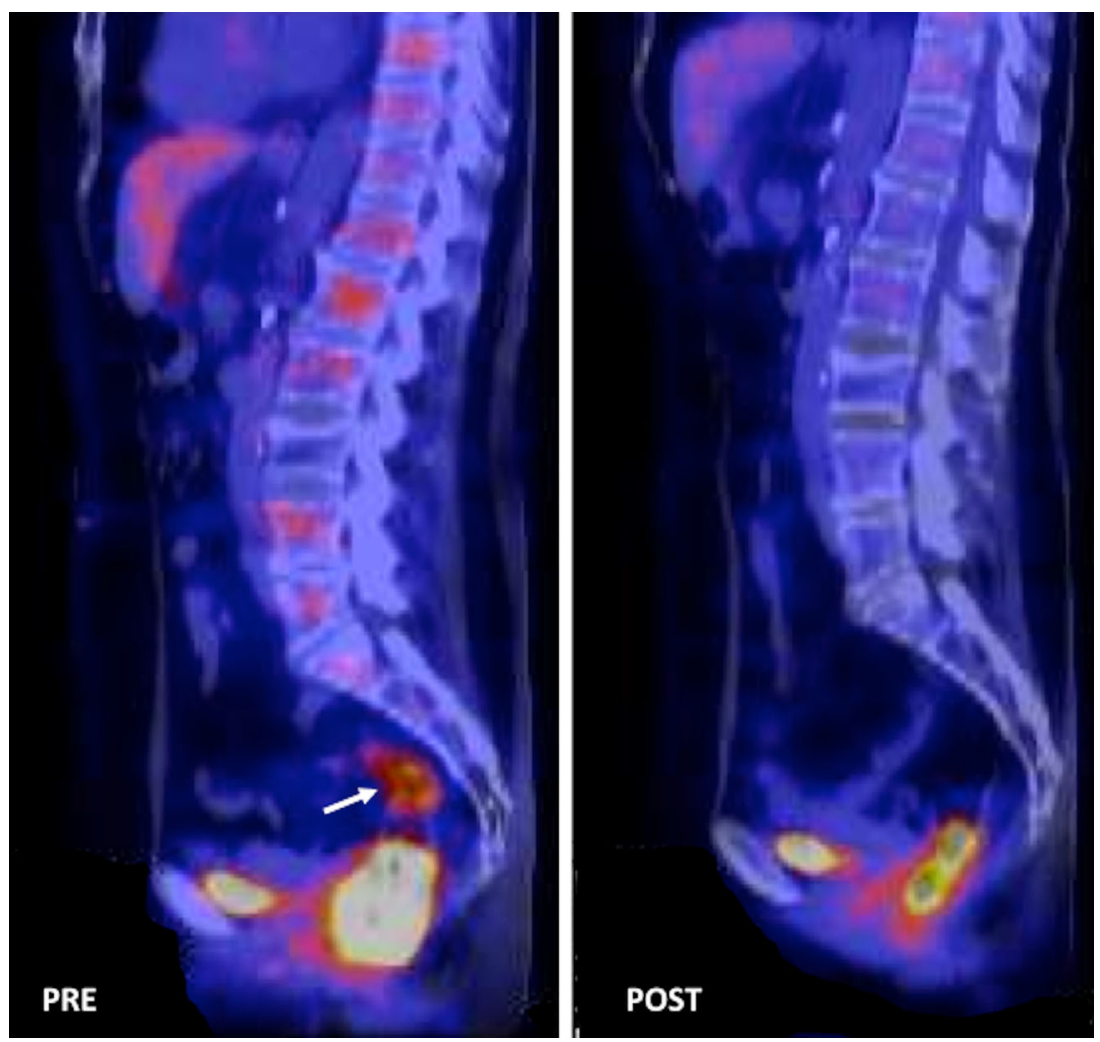


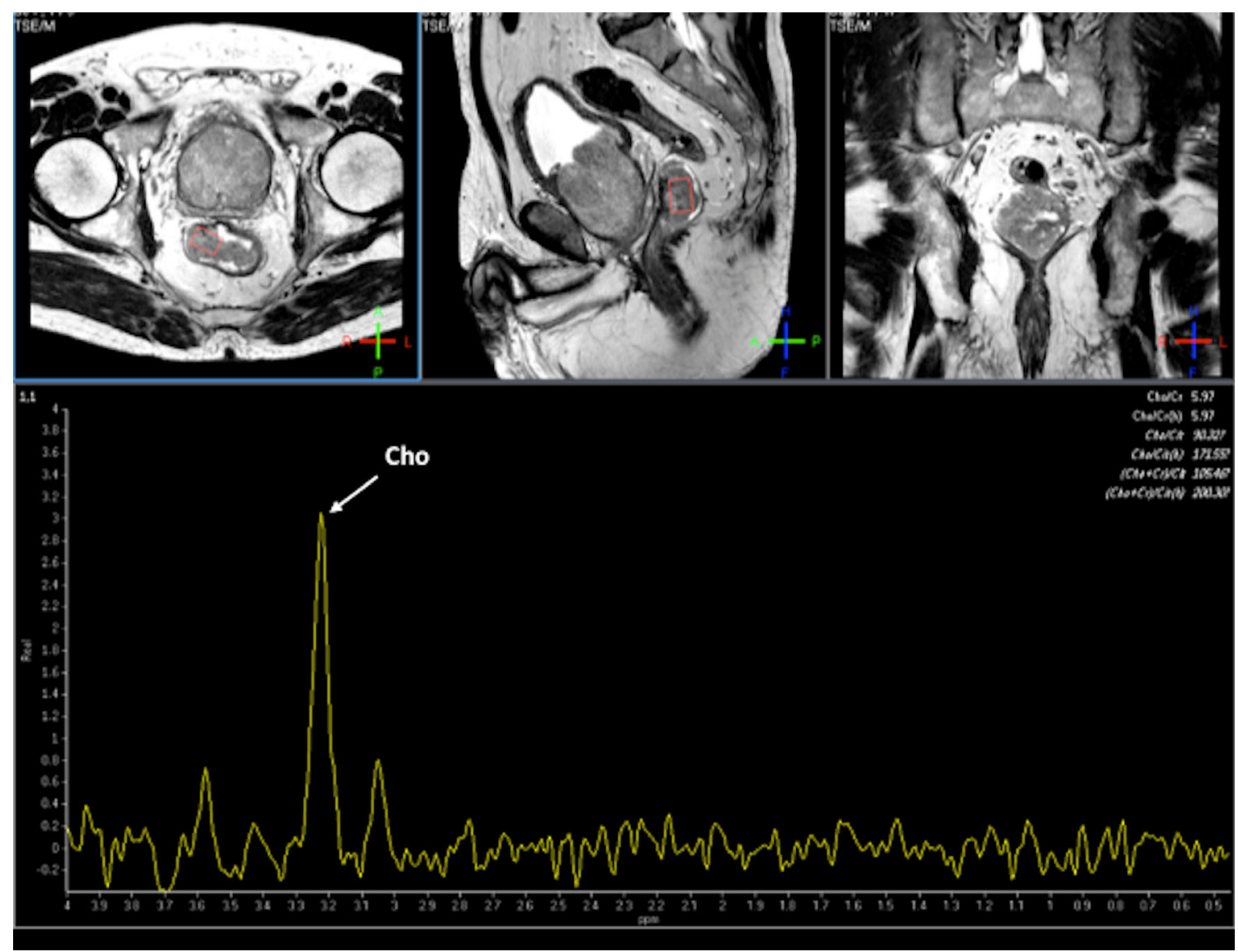

Fig. 12 MR spectroscopy of rectal polyp. Proton MRSI of a rectal polyp. Axial (upper row), sagittal (middle), and coronal (right) images obtained by T2-weighted turbo spin-echo imaging. Spectra obtained by univoxel spectroscopy at long echo time $(\mathrm{TE}=135 \mathrm{~ms})$ showed an increased level of choline (Cho) peak at $3.2 \mathrm{ppm}$

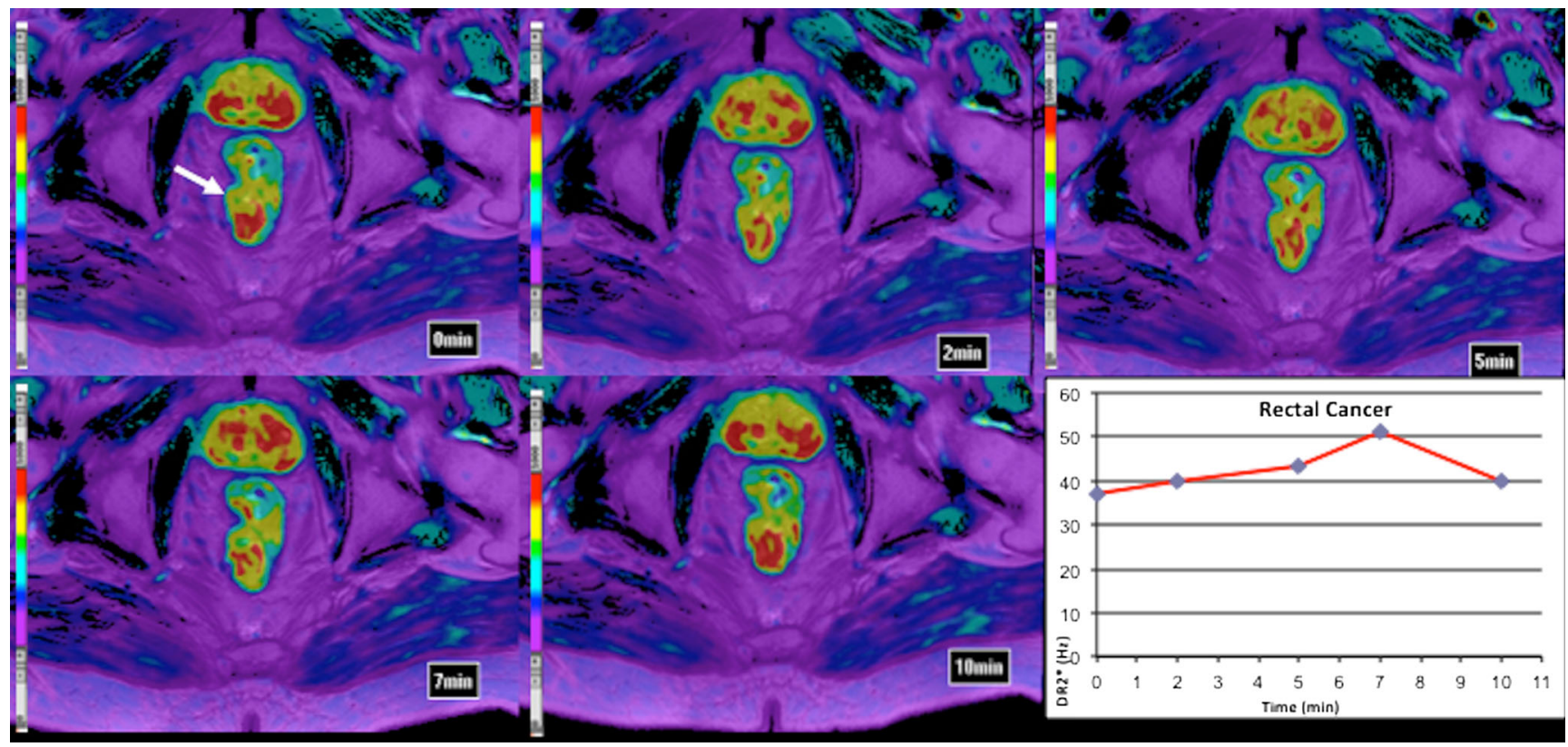

Fig. 13 Tumour hypoxia. BOLD images (fusion of T2 and T2*) pre- and following the administration of oxygen may demonstrate a mucinous adenocarcinoma of the rectum. In basal conditions, the tumour showed high signal on $\mathrm{T}_{2} *$ map. After $\mathrm{O}_{2}$ administration, the signal decreased with an ascending curve of $\Delta \mathrm{R} 2 *$ (red line), which is related to tumour radioresistance 
Fig. 14 MR lymphography. Metastatic 6-mm node (white arrow) on T2*-weighted highresolution specimen MRI with the histopathological correlation (right) in a patient with a pT3 $\mathrm{pN} 1$ moderately differentiated adenocarcinoma

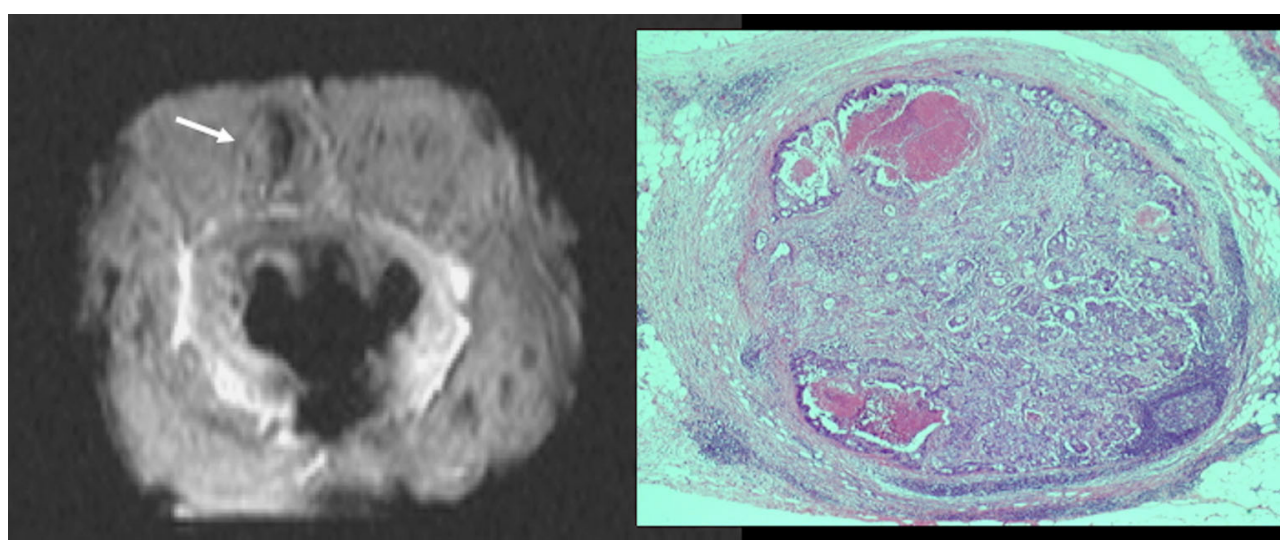

positron emission tomography (PET-CT) in the re-staging of RC after CRTP, which suggests that the major strength of imaging lies in the identification of non-responders. Both DWI and ${ }^{18}$ F-FDG PET-CT are not actually accurate enough to safely select patients for possible organ-sparing strategies [70]. In the case of tumour recurrence, a study by Lambregts et al. using DWI for diagnosing local tumour regrowth during follow-up of organ preservation treatment after CRTP for RC evidenced that although there was no overall improvement in diagnostic performance in terms of AUC, DWI improved the sensitivity of MRI for diagnosing local tumour regrowth and lowered the rate of equivocal MR exams [73].

Finally, we must consider that the standard monoexponential analysis of DWI assumes a Gaussian behavior of water diffusion. However, in many biological tissues, the presence of barriers (e.g., cell membranes and intracellular organelles) and compartments (intracellular, intravascular, and EES) alter the water diffusion process so that it is no longer Gaussian. The kurtosis is a dimensionless statistical metric for quantifying the non-gaussianity of diffusion. A large diffusional kurtosis suggests a high degree of diffusional heterogeneity and microstructural complexity, which is usually the case of tumour lesions (Fig. 5) [74]. Preliminary results in body imaging open the consideration of a future role for this imaging technique in RC [74].

\section{Molecular imaging of tumour proliferation with PET}

\footnotetext{
${ }^{18} \mathrm{~F}$-3-deoxy-3-fluorothymidine (FLT) is a radiotracer, which allows the evaluation of cellular proliferation. Nonetheless, FLT-PET evidenced a limited value in CRC, demonstrating less sensitivity than ${ }^{18} \mathrm{~F}$-FDG-PET for the detection of pathologic LNs or liver metastases in CRC $[75,76]$.
}

\section{Tumour metabolism in CRC}

Tumour proliferation needs nutrients, energy, and biosynthetic activity. This feature is responsible for the metabolic reprogramming associated with cancer. Imaging techniques allow assessment of the status of the altered metabolic pathways in CRC.

Imaging energetic metabolism with ${ }^{18} F-F D G$-PET

A well-known energy metabolism alteration in tumour cells is an increased glycolytic capacity, even in the presence of a high $\mathrm{O}_{2}$ concentration, a process named aerobic glycolysis. Proliferating tumour cells generally consume glucose at a high rate. This increased glucose uptake is the basis for clinical PET imaging in tumours. PET-CT is an established clinical technique for the management of CRC patients, which may have an impact on changing patient management strategies with its evolving role in diagnosis, radiation therapy planning, prediction of response, and therapy assessment [77-80]. PET-CT may upstage a significant proportion the patients in RC by identifying unsuspected systemic or LN metastases [77, 78]. Nonetheless, routine PET for the initial staging of CRC is not an established indication of the technique. PET may also help to guide decisions concerning metastasectomy for patients with CRC by excluding unresectable metastatic disease [77]. Some papers support the accuracy of PET in predicting $\mathrm{pCR}$, while others dispute its utility [70, 81-83] (Figs. 10 and 11). However, PET will likely not have the ability to detect patients with pathologic evidence of very few tumour cells in fibrotic tissue in the surgical specimen after CRTP [70]. A metaanalysis established that complete metabolic response on ${ }^{18} \mathrm{~F}$ FDG-PET data after preoperative CRT is predictive of OS in $\mathrm{RC}$ [84]. Finally, PET is also more sensitive than conventional imaging $(\mathrm{CT})$ in detecting tumour relapse. CT scans were positive in $82 \%$ of patients and ${ }^{18}$ F-FDG-PET-CT in $98 \%$ of patients [85]. However, several limitations of PET for the evaluation of CRC must be considered. ${ }^{18} \mathrm{~F}-\mathrm{FDG}$ uptake is dependent on tumour grade and histological type, and PET shows a limited and a poor spatial resolution (missing small lesions).

PET-MRI has recently become available for clinical use. Combined anatomic and functional capabilities of 
MR imaging and the metabolic information of PET provide new insight into tumour phenotypes at a lower radiation dose than a PET-CT. Current literature is sparse concerning the role of PET-MRI in CRC [86]. Perhaps this hybrid technique might prove valuable in offering an increased confidence in the evaluation of liver lesions and residual masses after treatment, and improving identification of LNs.

\section{MR spectroscopy for evaluating metabolism}

There has been scarce literature published on the use of proton $\left({ }^{1} \mathrm{H}\right)$ MR spectroscopy (MRS) in CRC. Kim et al. demonstrated that RC mainly showed elevated choline (Cho) at $3.2 \mathrm{ppm}$ and lipids (Lip) peaks at $1.3 \mathrm{ppm}$ on MRS (Fig. 12). After CRTP, the Cho peak disappeared, resulting in only the Lip peak [87]. MRS has also been found of interest in the

a

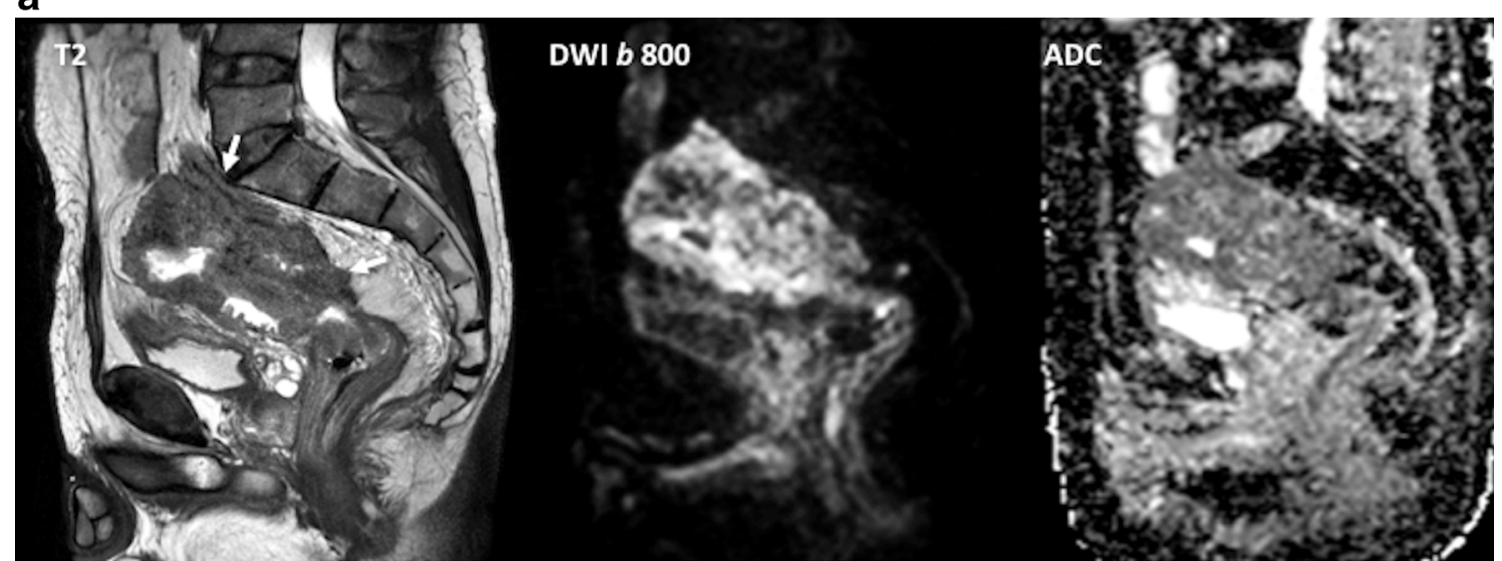

b
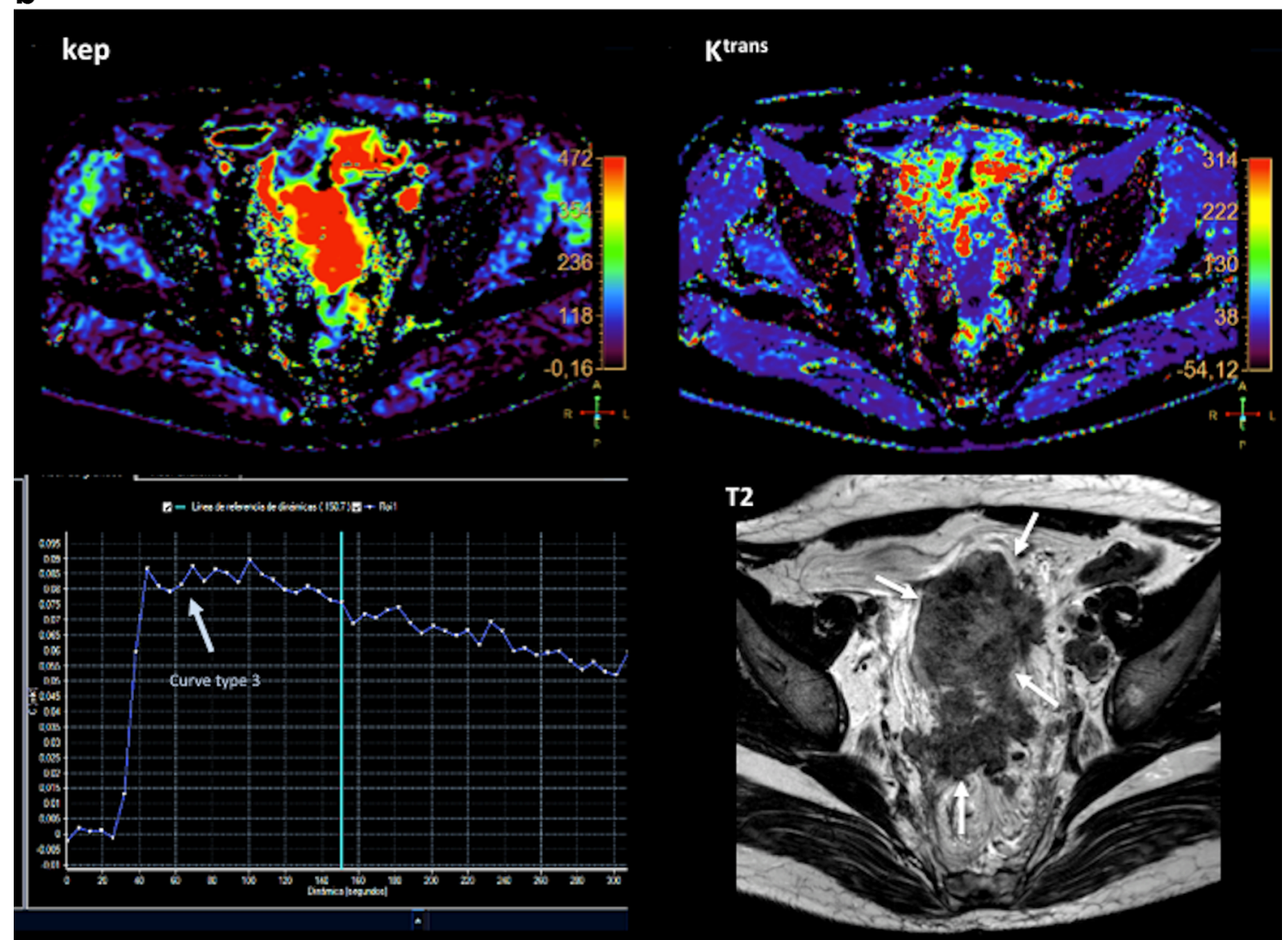

Fig. 15 Multiparametric evaluation of a 64-year-old patient with rectal cancer, pre-therapy. (a) Sagittal fast spin-echo T2-weighted image (left) and sagittal diffusion-weighted image with high $b$-value $\left(b=800 \mathrm{~s} / \mathrm{mm}^{2}\right)$ (center) and ADC map showed a big rectal tumour with restricted diffusion. (b) Perfusion MRI-related parametric maps (transfer constant, $\mathrm{K}^{\text {trans }}$

and return constant, $\mathrm{k}_{\mathrm{ep}}$ ), gadolinium concentration/time curve of the tumour evidenced increased perfusion within the tumour and a type 3 curve. (c) MR spectroscopy depicted an increased lipids peak. (d) Sagittal ${ }^{18}$ F-FDG-PET image evidenced an increased uptake of glucose in the tumour (red arrows) 
diagnosis of postsurgical recurrence of RC. An increase of residual Lip peaks in the postsurgical bed suggests tumour recurrence, while lower Lip peaks are present in scarring postoperative fibrosis [88]. However, MRS shows no clear clinical application in CRC because MRS evaluation of these tumours is technically demanding, not only for bowel movement but also for the presence of multiple interfaces between air, rectal wall, and perirectal fat that may result in an inhomogeneous magnetic field and poor spectra quality.

\section{Imaging oxygenation and hypoxia in CRC}

Hypoxia is considered to be an important mediator of malignant disease progression that has an important role in predicting the response to radiotherapy and an impact on patient prognosis and survival in CRC. To our knowledge, imaging evaluation of hypoxia in CRC has been scarce in published literature. Blood oxygenation level-dependent (BOLD)-MRI may provide a non-invasive means of assessing in-vivo tumour oxygenation based on endogenous deoxyhemoglobin as a contrast agent (Fig. 13). However, BOLD-MRI shows several limitations that need to be considered. First, no correlation between BOLD-MRI measurements and hypoxic markers has been published in CRC. Second, this technique is more likely to reflect acute tissue hypoxia (perfusion-related and often transient) than chronic (caused by increased oxygen diffusion distances due to tumour expansion) hypoxia and requires the simultaneous assessment of the functionality of tumour vasculature. And third, motion and susceptibility artifacts limit the clinical application of BOLD-MRI in CRC [89]. There is also a limited experience with the PET evaluation of hypoxia in CRC using hypoxiarelated radiotracers in $\mathrm{CRC}$ [75].

\section{Functional imaging of lymph nodes}

Nodal metastases are one of the most significant indicators of local recurrence and cancer-specific mortality in RC patients, and influence the determination of surgical and adjuvant treatments. CT, MRI, and endorectal ultrasound lacked sufficient accuracy to identify metastatic LNs with sensitivities and specificities in the 55-78\% range. Apart from this, histological data show that up to $45 \%$ of nodal metastases in RC are $\leq 4 \mathrm{~mm}$, which increases the difficulty of the accurate characterization of LNs. The development of ultrasmall iron oxide particle (USPIO) contrast agents opened the possibility of performing MR lymphography (MRL) [90]. The contrast agent shows a specific cellular uptake by the macrophages in normally functioning nodes. Macrophage sequestration within normal LNs causes decreases in nodal signal intensity c
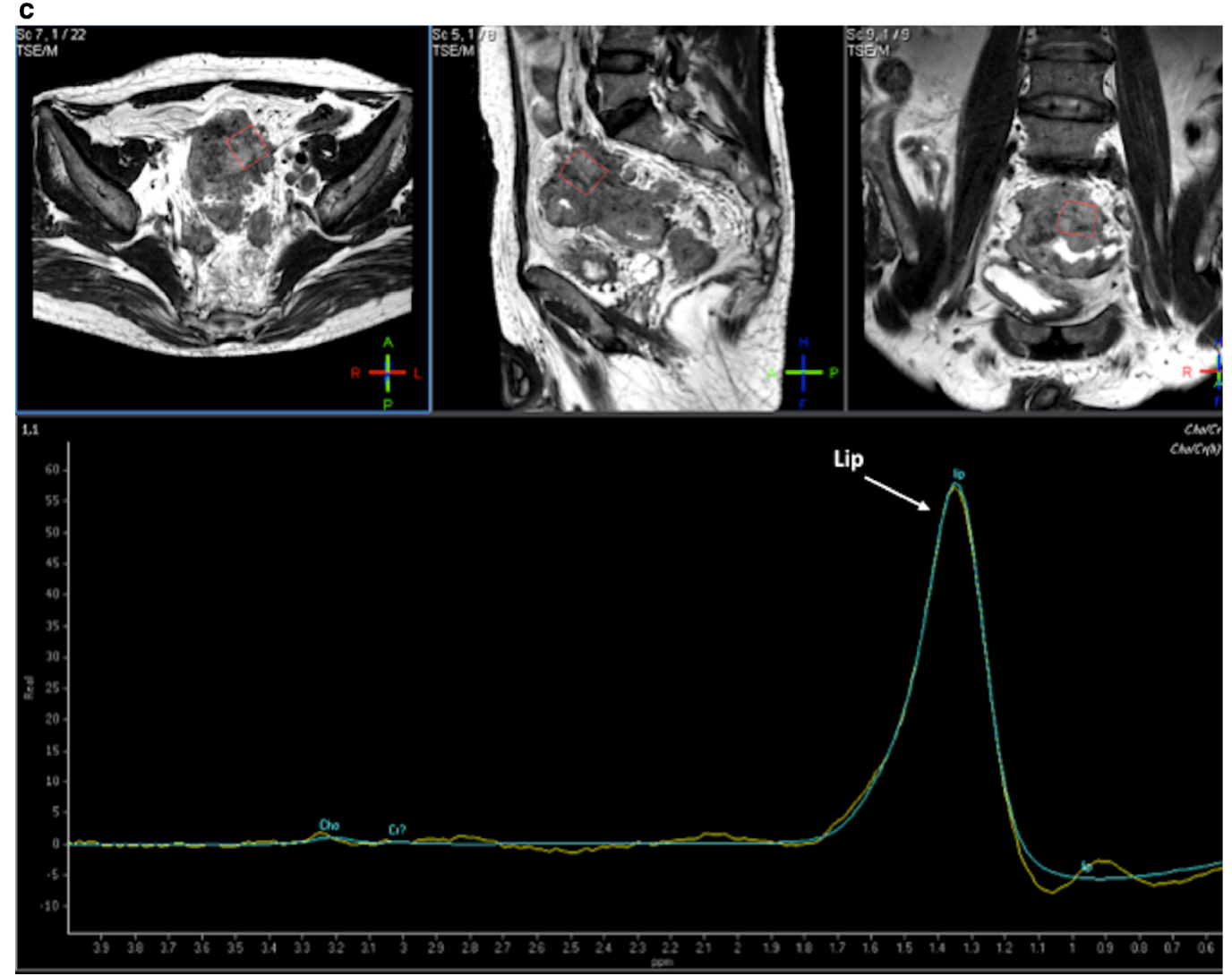

Fig. 15 (continued)

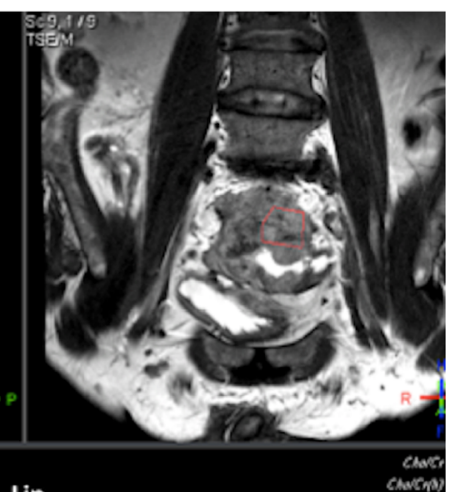

d

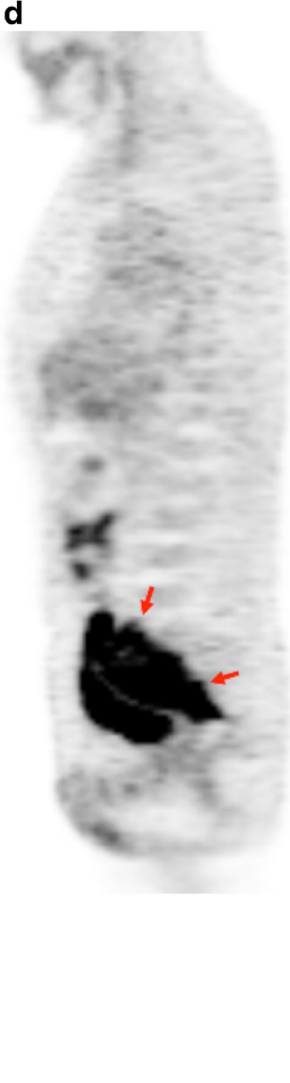


(SI) on susceptibility-weighted (T2*) MRI, whereas infiltrated LNs do not have macrophages and cannot take up the contrast agent (Fig. 14). MRL has demonstrated a sensitivity of $93 \%$ and a specificity of $96 \%$ for nodal staging in RC [91]. However, several limitations must be considered when we evaluated these data. First, MRI has a limited spatial resolution, meaning that only nodes that could be directly co-located between pre-operative in-vivo images and histopathology analysis were evaluated in published papers, thus excluding many other LNs (seen at pathology but not by MRI, and vice versa). This fact necessarily introduces a bias toward the assessment of larger LNs, which are naturally more visible by imaging. Second, an overlap of SI between benign and malignant LNs has been reported due to different features, including partial volume and lipomatosis. Finally, the evaluation of numerous LNs is a complex and time-consuming activity.

a

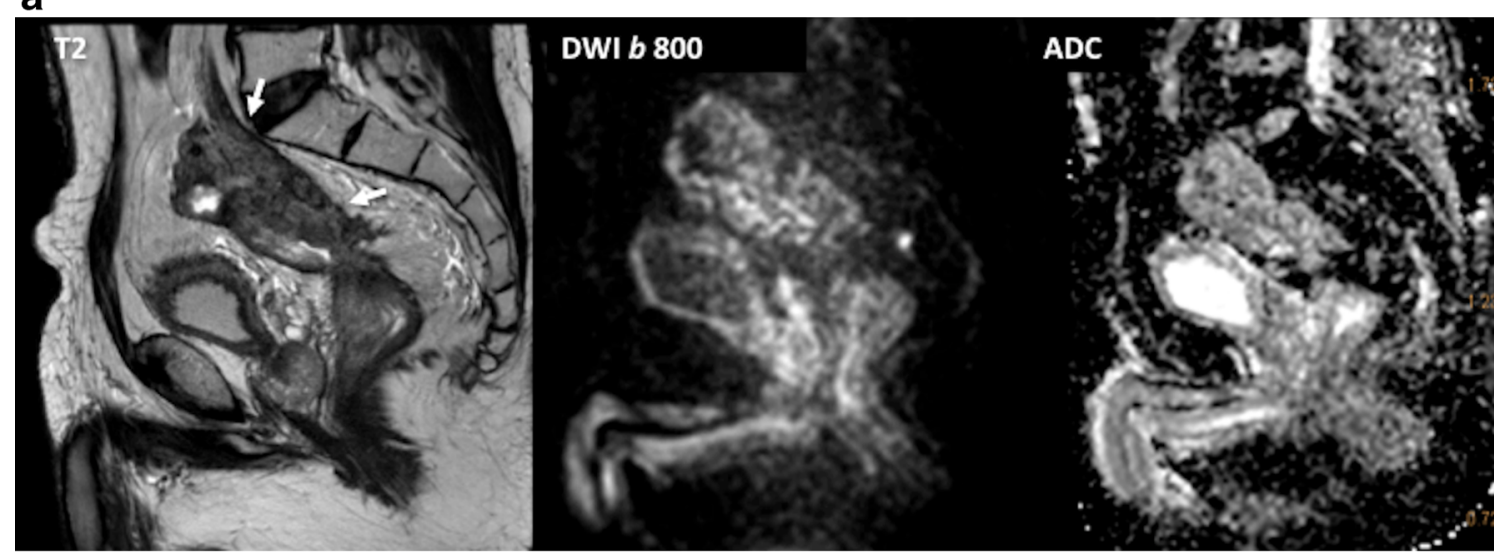

b

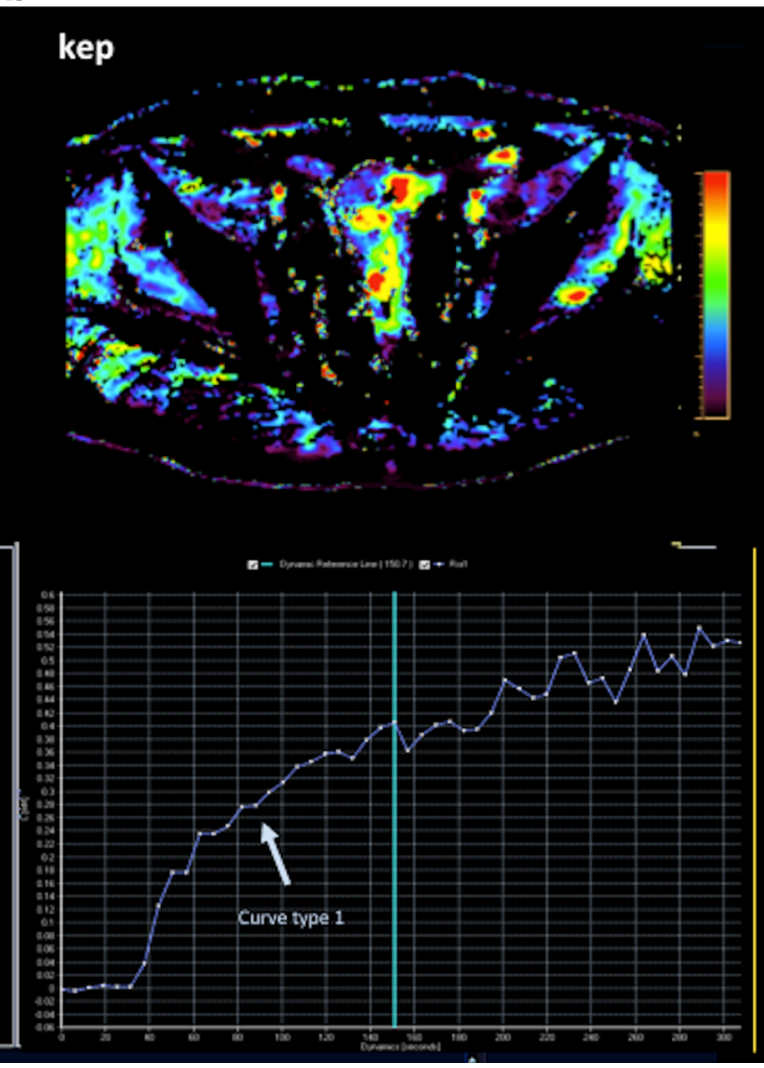

$\mathbf{K}^{\text {trans }}$
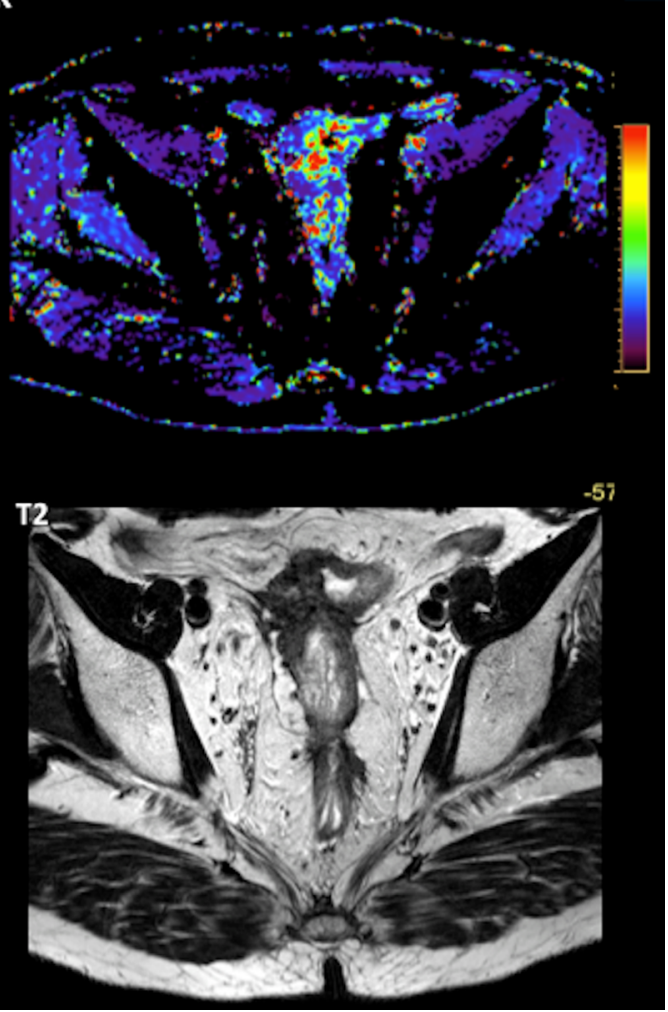

Fig. 16 Multiparametric evaluation of a 64-year-old patient with rectal cancer, post-therapy. (a) Sagittal fast spin-echo T2-weighted image (left), sagittal diffusion-weighted image with high $b$-value $\left(b=800 \mathrm{~s} / \mathrm{mm}^{2}\right)$ (center), and ADC map showed a reduction of tumour volume and increased ADC values. (b) Perfusion MRI-related parametric maps (transfer constant, $\mathrm{K}^{\text {trans }}$ and return constant, kep) and gadolinium

concentration/time curve of the tumour evidenced decreased perfusion within the tumour and a change in the type of curve (type 1 curve). (c) MR spectroscopy did not depict any metabolite peak. (d) Sagittal ${ }^{18} \mathrm{~F}$ FDG-PET image evidenced a decreased uptake of glucose in the tumour (red arrow). All these features indicated partial tumour response 
Perhaps the use of USPIO combined with DWI may simplify the process and allow for more accurate detection of nodal metastases [92]. However, to date, the use of USPIO agents in clinical practice is not possible.

The use of gadofosveset (a gadolinium-based MRI contrast agent, which acts as a blood pool agent) can also significantly improve the diagnostic performance to discriminate between benign and metastatic LNs in RC. The intravenous administration of this contrast media results in a selective uptake of contrast in benign LNs, which causes an increase in signal on T1-weighted images, and enhances the chemical shift artfact around the border of the nodes [93].

\section{Multiparametric evaluation of CRC}

Combining the anatomical resolution of imaging with functional (such as DWI and DCE imaging) and molecular (PET) techniques may offer additional information about treatment of tumour microenvironments in different clinical scenarios, including staging, tumour characterization, prediction of response to treatment, and response evaluation [94-101]. The use of these techniques allow for the quantitative evaluation of tumour phenotype, including changes in tumour biology that occur after therapy (Figs. 15 and 16). There has been a very limited use of the multiparametric/multimodality approach in CRC. These studies are mainly confined to experimental settings, and most of them have been single-center studies with small numbers of patients. The relationship between different parameters may explain tumour biological features. In this setting, CRC with a low-flow/high-metabolism phenotype demonstrated higher vascular endothelial growth factor (VEGF) expression and may reflect a more angiogenic and aggressive phenotype [98]. Fischer et al. reported that changes in the flow-metabolic phenotype (blood flow $\times$ maximum standardized uptake values (SUV)) of RC after CRT showed high accuracy for the prediction of histopathological response to CRTP (AUC 0.955, $95 \%$ confidence interval 0.833-1.000) using a cut-off value of $-75 \%$ [101]. However, at present, there is no standardized imaging protocol for multiparametric

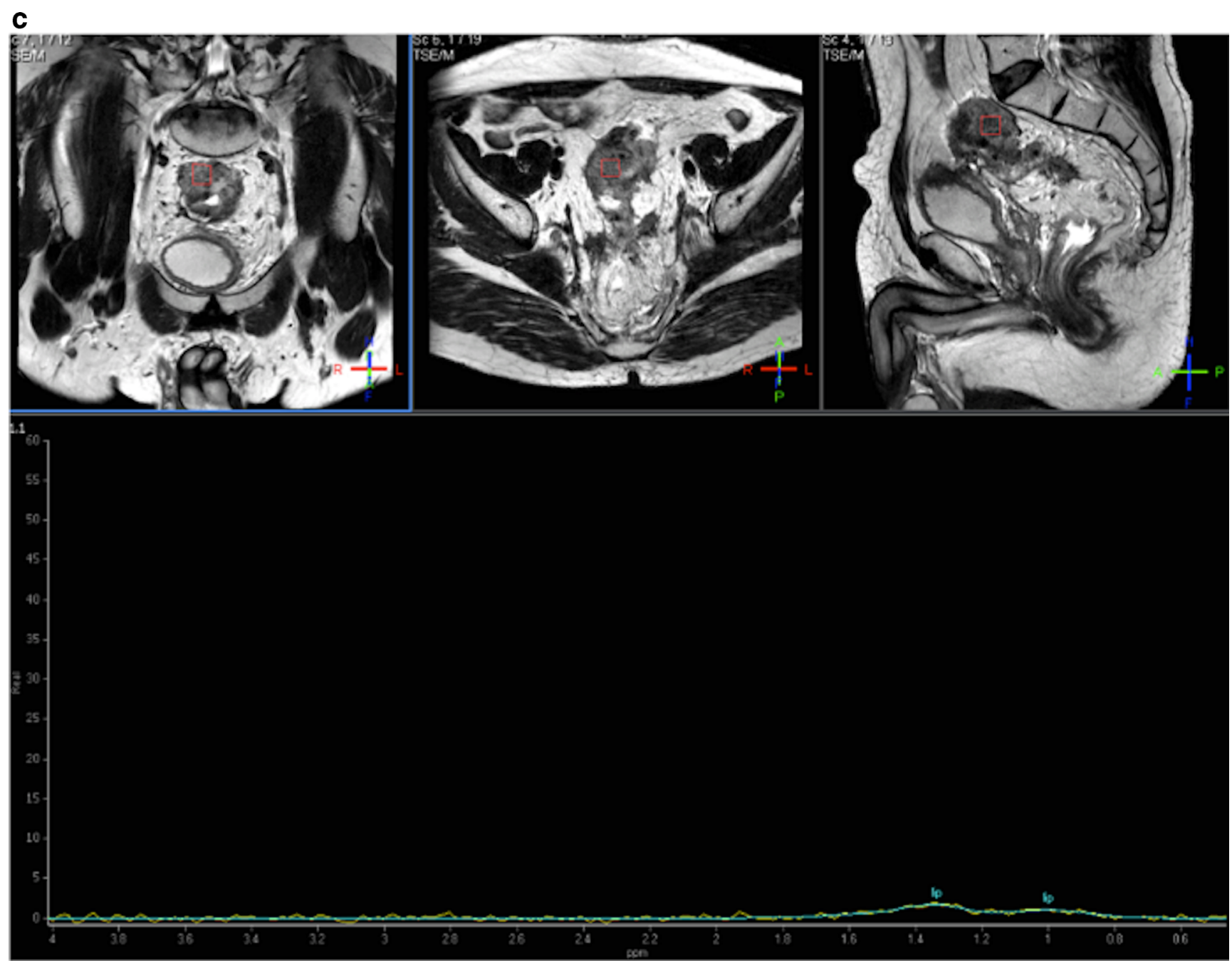

Fig. 16 (continued) 


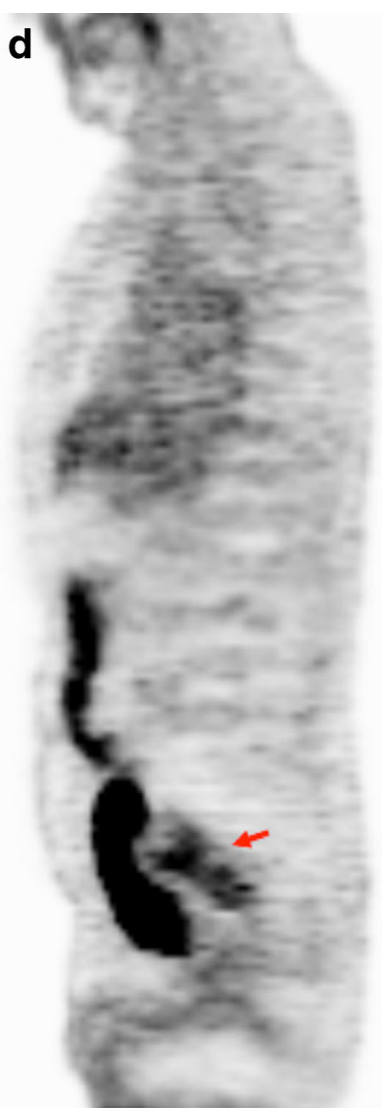

Fig. 16 (continued)

imaging evaluation in CRC patients, which complicates its clinical implementation.

\section{Conclusion}

In conclusion, advanced imaging techniques offer great opportunities in the evaluation of patients with CRC. The introduction of functional and molecular imaging techniques in clinical practice allows for the assessment of tumour hallmarks and tumour heterogeneity, which may change the management and therefore the prognosis of patients.

Open Access This article is distributed under the terms of the Creative Commons Attribution 4.0 International License (http:// creativecommons.org/licenses/by/4.0/), which permits unrestricted use, distribution, and reproduction in any medium, provided you give appropriate credit to the original author(s) and the source, provide a link to the Creative Commons license, and indicate if changes were made.

\section{References}

1. De Divitiis C, Nasti G, Montano M, Fisichella R, Iaffaioli RV, Berretta M (2014) Prognostic and predictive response factors in colorectal cancer patients: Between hope and reality. World J Gastroenterol 20:15049-15059

2. Kekelidze M, D'Errico L, Pansini M, Tyndall A, Hohmann J (2013) Colorectal cancer: Current imaging methods and future perspectives for the diagnosis, staging and therapeutic response evaluation. World J Gastroenterol 19:8502-8514

3. McQueen AS, Scott J (2012) CT staging of colorectal cancer: What do you find in the chest? Clin Radiol 4:352-358

4. Lim GH, Koh DC, Cheong WK, Wong KS, Tsang CB (2009) Natural history of small, "indeterminate" hepatic lesions in patients with colorectal cancer. Dis Colon Rectum 52:1487-1491

5. Hanahan D, Weinberg RA (2011) Hallmarks of cancer: The next generation. Cell 144:646-674

6. Kapse N, Goh V (2009) Functional imaging of colorectal cancer: Positron emission tomography, magnetic resonance imaging, and computed tomography. Clin Colorectal Cancer 8:77-87

7. Figueiras RG, Goh V, Padhani AR, Naveira AB, Caamaño AG, Martin CV (2010) The role of functional imaging in colorectal cancer. AJR Am J Roentgenol 195:54-66

8. Prezzi D, Goh V (2015) Rectal cancer magnetic resonance imaging: Imaging beyond morphology. Clin Oncol (R Coll Radiol). doi:10.1016/j.clon.2015.10.010

9. Torkzad MR, Påhlman L, Glimelius B (2007) Magnetic resonance imaging (MRI) in rectal cancer: A comprehensive review. Insights Imaging 1:245-267

10. Spada C, Stoker J, Alarcon O et al (2015) Clinical indications for computed tomographic colonography: European Society of Gastrointestinal Endoscopy (ESGE) and European Society of Gastrointestinal and Abdominal Radiology (ESGAR) guideline. Eur Radiol 25:331-345

11. Laghi A (2014) Computed tomography colonography in 2014: An update on technique and indications. World J Gastroenterol 20: 16858-16867

12. de Haan M, Pickhardt PJ, Stoker J (2015) CT colonography: Accuracy, acceptance, safety and position in organised population screening. Gut 64:342-350

13. ACR-SAR-SCBT-MR practice parameter for the performance of computed tomography (CT) colonography in adults. Available at: http://www.acr.org/ / media/ A81531ACA92F45058A83B5281E8FE826.pdf. Accesed July 25, 2015

14. Kornprat P, Pollheimer MJ, Lindtner RA, Schlemmer A, Rehak P, Langner C (2011) Value of tumor size as a prognostic variable in colorectal cancer: A critical reappraisal. Am J Clin Oncol 34:43-49

15. Balta AZ, Özdemir Y, Sücüllü İ et al (2014) Can horizontal diameter of colorectal tumor help predict prognosis? Ulus Cerrahi Derg 30:115-119

16. Todosi A, Huțanu I, Gavrilescu MM et al (2015) Assessment of tumor parameters as factors of aggressiveness in colon cancer. J Surg [Jurnalul de Chirurgie] 10:271-275

17. Neri E, Guidi E, Pancrazi F et al (2015) MRI tumor volume reduction rate vs tumor regression grade in the pre-operative restaging of locally advanced rectal cancer after chemo-radiotherapy. Eur J Radiol. doi:10.1016/j.ejrad.2015.08.008

18. Kang JH, Kim YC, Kim H et al (2010) Tumor volume changes assessed by three-dimensional magnetic resonance volumetry in rectal cancer patients after preoperative chemoradiation: The impact of the volume reduction ratio on the prediction of pathologic complete response. Int J Radiat Oncol Biol Phys 76:1018-1025

19. Petrillo M, Fusco R, Catalano O et al (2015) MRI for assessing response to neoadjuvant therapy in locally advanced rectal cancer using DCE-MR and DW-MR data sets: A preliminary report. Biomed Res Int. doi:10.1155/2015/514740 
20. Adams RB, Aloia TA, Loyer E et al (2013) Selection for hepatic resection of colorectal liver metastases: Expert consensus statement. HPB (Oxford) 15:91-103

21. Frankel TL, Gian RK, Jarnagin WR (2012) Preoperative imaging for hepatic resection of colorectal cancer metastasis. J Gastrointest Oncol 3:11-18

22. Lim MC, Tan CH, Cai J, Zheng J, Kow AW (2014) CT volumetry of the liver: Where does it stand in clinical practice? Clin Radiol 69:887-895

23. Torkzad MR, Norén A, Kullberg J (2012) Stereology: A novel technique for rapid assessment of liver volume. Insights Imaging 3:387-393

24. Boellaard TN, Henneman OD, Streekstra GJ et al (2013) The feasibility of colorectal cancer detection using dual-energy computed tomography with iodine mapping. Clin Radiol 68:799-806

25. Kato T, Uehara K, Ishigaki S et al (2015) Clinical significance of dual-energy CT-derived iodine quantification in the diagnosis of metastatic LN in colorectal cancer. Eur J Surg Oncol 41: $1464-1470$

26. Liu H, Yan F, Pan Z et al (2015) Evaluation of dual energy spectral $\mathrm{CT}$ in differentiating metastatic from non-metastatic lymph nodes in rectal cancer: initial experience. Eur J Radiol 84:228-234

27. Davnall F, Yip CS, Ljungqvist $\mathrm{G}$ et al (2012) Assessment of tumor heterogeneity: An emerging imaging tool for clinical practice? Insights Imaging 2012(3):573-589

28. Cui C, Cai H, Liu L, Li L, Tian H, Li L (2011) Quantitative analysis and prediction of regional lymph node status in rectal cancer based on computed tomography imaging. Eur Radiol 21: 2318-2325

29. Ng F, Ganeshan B, Kozarski R, Miles KA, Goh V (2013) Assessment of primary colorectal cancer heterogeneity by using whole-tumor texture analysis: contrast-enhanced CT texture as a biomarker of 5-year survival. Radiology 266:177-184

30. Lubner MG, Stabo N, Lubner SJ et al (2015) CT textural analysis of hepatic metastatic colorectal cancer: Pre-treatment tumor heterogeneity correlates with pathology and clinical outcomes. Abdom Imaging 40:2331-2337

31. García-Figueiras R, Padhani AR, Beer AJ et al (2015) Imaging of tumor angiogenesis for radiologists-Part 1: Biological and technical basis. Curr Probl Diagn Radiol 44:407-424

32. Kierkels RG, Backes WH, Janssen MH et al (2010) Comparison between perfusion computed tomography and dynamic contrastenhanced magnetic resonance imaging in rectal cancer. Int $\mathrm{J}$ Radiat Oncol Biol Phys 77:400-408

33. Sahani DV, Kalva SP, Hamberg LM et al (2005) Assessing tumor perfusion and treatment response in rectal cancer with multisection CT: Initial observations. Radiology 234:785-792

34. Goh V, Glynne-Jones R (2014) Perfusion CT imaging of colorectal cancer. Br J Radiol 87:20130811

35. García-Figueiras R, Goh VJ, Padhani AR et al (2013) CT perfusion in oncologic imaging: A useful tool? AJR Am J Roentgenol 200:8-19

36. Kim YE, Lim JS, Choi J et al (2013) Perfusion parameters of dynamic contrast-enhanced magnetic resonance imaging in patients with rectal cancer: Correlation with microvascular density and vascular endothelial growth factor expression. Korean J Radiol 14:878-885

37. Hong HS, Kim SH, Choi J et al (2013) Correlations of dynamic contrast-enhanced magnetic resonance imaging with morphologic, angiogenic, and molecular prognostic factors in rectal cancer. Yonsei Med J 54:123-130

38. Lim JS, Kim D, Baek SE et al (2012) Perfusion MRI for the prediction of treatment response after preoperative chemoradiotherapy in locally advanced rectal cancer. Eur Radiol 22:1693-1700
39. Gollub MJ, Gultekin DH, Akin O et al (2012) Dynamic contrast enhanced-MRI for the detection of pathological complete response to neoadjuvant chemotherapy for locally advanced rectal cancer. Eur Radiol 22:821-831

40. Martens MH, Subhani S, Heijnen LA et al (2015) Can perfusion MRI predict response to preoperative treatment in rectal cancer? Radiother Oncol 114:218-223

41. Intven M, Reerink O, Philippens ME (2015) Dynamic contrast enhanced MR imaging for rectal cancer response assessment after neo-adjuvant chemoradiation. J Magn Reson Imaging 41: $1646-1653$

42. Tong T, Sun Y, Gollub MJ et al (2015) Dynamic contrastenhanced MRI: Use in predicting pathological complete response to neoadjuvant chemoradiation in locally advanced rectal cancer. J Magn Reson Imaging. doi:10.1002/jmri.24835

43. Gollub MJ, Cao K, Gollub MJ et al (2013) Prognostic aspects of DCE-MRI in recurrent rectal cancer. Eur Radiol 42:673-680

44. Beuzit L, Eliat PA, Brun V et al (2015) Dynamic contrastenhanced MRI: Study of inter-software accuracy and reproducibility using simulated and clinical data. J Magn Reson Imaging. doi:10.1002/jmri.25101

45. Bäuerle T, Seyler L, Münter M et al (2013) Diffusion-weighted imaging in rectal carcinoma patients without and after chemoradiotherapy: A comparative study with histology. Eur J Radiol 82: 444-452

46. Ganten MK, Schuessler M, Bäuerle T et al (2013) The role of perfusion effects in monitoring of chemoradiotherapy of rectal carcinoma using diffusion-weighted imaging. Cancer Imaging 13:548-556

47. García-Figueiras R, Padhani AR, Beer AJ et al (2015) Imaging of tumor angiogenesis for radiologist part 2: Clinical utility. Curr Probl Diagn Radiol 44:425-436

48. Sun H, Xu Y, Yang Q, Wang W (2014) Assessment of tumor grade and angiogenesis in colorectal cancer: Whole-volume perfusion CT. Acad Radiol 21:750-757

49. Kim JW, Jeong YY, Chang NK et al (2012) Perfusion CT in colorectal cancer: Comparison of perfusion parameters with tumor grade and microvessel density. Korean J Radiol 13(Suppl 1):S89-S97

50. Hayano K, Fujishiro T, Sahani DV et al (2014) Computed tomography perfusion imaging as a potential imaging biomarker of colorectal cancer. World J Gastroenterol 20:17345-17351

51. Prezzi D, Khan A, Goh V (2015) Perfusion CT imaging of treatment response in oncology. Eur J Radiol. doi:10.1016/j.ejrad. 2015.03.022

52. Janssen MH, Aerts HJ, Kierkels RG et al (2010) Tumor perfusion increases during hypofractionated short-course radiotherapy in rectal cancer: Sequential perfusion-CT findings. Radiother Oncol 94:156-160

53. Willett CG, Duda DG, di Tomaso E et al (2009) Efficacy, safety, and biomarkers of neoadjuvant bevacizumab, radiation therapy, and fluorouracil in rectal cancer: A multidisciplinary phase II study. J Clin Oncol 27:3020-3026

54. Anzidei M, Napoli A, Zaccagna F et al (2011) Liver metastases from colorectal cancer treated with conventional and antiangiogenetic chemotherapy: Evaluation with liver computed tomography perfusion and magnetic resonance diffusionweighted imaging. J Comput Assist Tomogr 35:690-696

55. Padhani AR, Liu G, Koh DM et al (2009) Diffusion-weighted magnetic resonance imaging as a cancer biomarker: Consensus and recommendations. Neoplasia 11:102-125

56. Ichikawa T, Erturk SM, Motosugi U et al (2006) High-B-value diffusion weighted MRI in colorectal cancer. AJR Am J Roentgenol 187:181-184

57. Heijnen LA, Lambregts DM, Mondal D et al (2013) Diffusionweighted MR imaging in primary rectal cancer staging 
demonstrates but does not characterise lymph nodes. Eur Radiol 23:3354-3360

58. Kim SH, Yoon JH, Lee Y (2015) Added value of morphologic characteristics on diffusion-weighted images for characterizing lymph nodes in primary rectal cancer. Clin Imaging 39: 1046-1051

59. Cho EY, Kim SH, Yoon JH et al (2013) Apparent diffusion coefficient for discriminating metastatic from non-metastatic lymph nodes in primary rectal cancer. Eur J Radiol 82:e662-e668

60. Lambregts DM, Maas M, Cappendijk VC et al (2011) Wholebody diffusion-weighted magnetic resonance imaging: Current evidence in oncology and potential role in colorectal cancer staging. Eur J Cancer 47:2107-2116

61. Gong J, Cao W, Zhang Z et al (2015) Diagnostic efficacy of whole-body diffusion-weighted imaging in the detection of tumour recurrence and metastasis by comparison with $18 \mathrm{~F}-2$ fluoro-2-deoxy-D-glucose positron emission tomography or computed tomography in patients with gastrointestinal cancer. Gastroenterol Rep (Oxford) 3:128-135

62. Sun Y, Tong T, Cai S, Bi R, Xin C, Gu Y (2014) Apparent Diffusion Coefficient (ADC) value: A potential imaging biomarker that reflects the biological features of rectal cancer. PLoS One 9: e109371

63. Song I, Kim SH, Lee SJ, Choi JY, Kim MJ, Rhim H (2012) Value of diffusion-weighted imaging in the detection of viable tumour after neoadjuvant chemoradiation therapy in patients with locally advanced rectal cancer: Comparison with T2 weighted and PET/ CT imaging. Br J Radiol 85:577-586

64. Jung SH, Heo SH, Kim JW et al (2012) Predicting response to neoadjuvant chemoradiation therapy in locally advanced rectal cancer: Diffusion-weighted 3 Tesla MR imaging. J Magn Reson Imaging 35:110-116

65. Barbaro B, Vitale R, Valentini V et al (2012) Diffusion-weighted magnetic resonance imaging in monitoring rectal cancer response to neoadjuvant chemoradiotherapy. Int J Radiat Oncol Biol Phys 83:594-959

66. Padhani AR, Koh DM (2011) Diffusion MR imaging for monitoring of treatment response. Magn Reson Imaging Clin N Am 19: 181-209

67. Park MJ, Kim SH, Lee SJ, Jang KM, Rhim H (2011) Locally advanced rectal cancer: added value of diffusion-weighted MR imaging for predicting tumor clearance of the mesorectal fascia after neoadjuvant chemotherapy and radiation therapy. Radiology 260:771-780

68. Curvo-Semedo L, Lambregts DM, Maas M et al (2011) Rectal cancer: assessment of complete response to preoperative combined radiation therapy with chemotherapy - conventional MR volumetry versus diffusion-weighted MR imaging. Radiology 60:734-743

69. Cho SH, Kim GC, Jang YJ et al (2014) Locally advanced rectal cancer: Post-chemoradiotherapy ADC histogram analysis for predicting a complete response. Acta Radiol 56:1042-1050

70. Joye I, Deroose CM, Vandecaveye V, Haustermans K (2014) The role of diffusion-weighted MRI and (18)F-FDG PET/CT in the prediction of pathologic complete response after radiochemotherapy for rectal cancer: A systematic review. Radiother Oncol 113: $158-165$

71. van der Paardt MP, Zagers MB, Beets-Tan RG, Stoker J, Bipat S (2013) Patients who undergo preoperative chemoradiotherapy for locally advanced rectal cancer restaged by using diagnostic MR imaging: A systematic review and meta-analysis. Radiology 269: $101-112$

72. Xie H, Sun T, Chen M et al (2015) Effectiveness of the apparent diffusion coefficient for predicting the response to chemoradiation therapy in locally advanced rectal cancer: A systematic review and meta-analysis. Medicine (Baltimore) 94(6):e517
73. Lambregts DM, Lahaye MJ, Heijnen LA et al (2015) MRI and diffusion-weighted MRI to diagnose a local tumour regrowth during long-term follow-up of rectal cancer patients treated with organ preservation after chemoradiotherapy. Eur Radiol

74. Rosenkrantz AB, Padhani AR, Chenevert TL et al (2015) Body diffusion kurtosis imaging: Basic principles, applications, and considerations for clinical practice. J Magn Reson Imaging 42: 1190-1202

75. Roels S, Slagmolen P, Nuyts J et al (2008) Biological image guided radiotherapy in rectal cancer: Is there a role for FMISO or FLT, next to FDG? Acta Oncol 47:1237-1248

76. Muijs CT, Beukema JC, Widder J et al (2011) 18F-FLT-PET for detection of rectal cancer. Radiother Oncol 98:357-359

77. Herbertson RA, Scarsbrook AF, Lee ST, Tebbutt N, Scott AM (2009) Established, emerging and future roles of PET/CT in the management of colorectal cancer. Clin Radiol 64:225-237

78. Schmoll HJ, Van Cutsem E, Stein A et al (2012) ESMO consensus guidelines for management of patients with colon and rectal cancer. A personalized approach to clinical decision making. Ann Oncol 23:2479-2516

79. Agarwal A, Marcus C, Xiao J, Nene P, Kachnic LA, Subramaniam RM (2014) FDG PET/CT in the management of colorectal and anal cancers. AJR Am J Roentgenol 203:1109-1119

80. Polat E, Bostanci EB, Aksoy E et al (2015) The impact of PET/CT on the management of hepatic and extra hepatic metastases from gastrointestinal cancers. Eur J Radiol 84:1165-1170

81. Guillem JG, Ruby JA, Leibold T et al (2013) Neither FDG-PET Nor CT can distinguish between a pathological complete response and an incomplete response after neoadjuvant chemoradiation in locally advanced rectal cancer: A prospective study. Ann Surg 258:289-295

82. Guerra L, Niespolo R, Di Pisa G et al (2011) Change in glucose metabolism measured by $18 \mathrm{~F}-\mathrm{FDG} \mathrm{PET} / \mathrm{CT}$ as a predictor of histopathologic response to neoadjuvant treatment in rectal cancer. Abdom Imaging 36:38-45

83. Maffione AM, Marzola MC, Capirci C, Colletti PM, Rubello D (2015) Value of (18)F-FDG PET for predicting response to neoadjuvant therapy in rectal cancer: Systematic review and metaanalysis. AJR Am J Roentgenol 204:1261-1268

84. Krug B, Crott R, de Cannière L, D'Hondt L, Vander Borght T (2013) A systematic review of the predictive value of $18 \mathrm{~F}$ fluoro-2-deoxyglucose positron emission tomography on survival in locally advanced rectal cancer after neoadjuvant chemoradiation. Color Dis 15:e627-e633

85. Deleau C, Buecher B, Rousseau C et al (2011) Clinical impact of fluorodeoxyglucose-positron emission tomography scan/ computed tomography in comparison with computed tomography on the detection of colorectal cancer recurrence. Eur J Gastroenterol Hepatol 23:275-281

86. Pace L, Nicolai E, Aiello M, Catalano OA, Salvatore M (2013) Whole-body PET/MRI in oncology: Current status and clinical applications. Clin Transl Imaging 1:31-44

87. Kim MJ, Lee SJ, Lee JH et al (2012) Detection of rectal cancer and response to concurrent chemoradiotherapy by proton magnetic resonance spectroscopy. Magn Reson Imaging 30:848-853

88. Jeon YS, Cho SG, Choi SK et al (2004) Differentiation of recurrent rectal cancer and postoperative fibrosis: Preliminary report by proton MR spectroscopy. J Korean Soc Magn Reson Med 8:24-31

89. Padhani AR, Krohn KA, Lewis JS, Alber M (2007) Imaging oxygenation of human tumours. Eur Radiol 17:861-872

90. Wu L, Cao Y, Liao C, Huang J, Gao F (2011) Diagnostic performance of USPIO-enhanced MRI for lymph-node metastases in different body regions: A meta-analysis. Eur J Radiol 80:582-589 
91. Lahaye MJ, Engelen SM, Kessels AG et al (2008) USPIOenhanced MR imaging for nodal staging in patients with primary rectal cancer: Predictive criteria. Radiology 246:804-811

92. Thoeny HC, Triantafyllou M, Birkhaeuser FD et al (2009) Combined ultrasmall superparamagnetic particles of iron oxideenhanced and diffusion-weighted magnetic resonance imaging reliably detect pelvic lymph node metastases in normal-sized nodes of bladder and prostate cancer patients. Eur Urol 55:761-769

93. Lambregts DM, Heijnen LA, Maas M et al (2013) Gadofosvesetenhanced MRI for the assessment of rectal cancer lymph nodes: Predictive criteria. Abdom Imaging 38:720-727

94. Attenberger UI, Pilz LR, Morelli JN et al (2014) Multi-parametric MRI of rectal cancer - do quantitative functional MR measurements correlate with radiologic and pathologic tumor stages? Eur J Radiol 83:1036-1043

95. Hötker AM, Garcia-Aguilar J, Gollub J (2014) Multiparametric MRI of rectal cancer in the assessment of response to therapy: A systematic review. Dis Colon Rectum 57:790-799

96. Heo SH, Kim JW, Shin SS, Jeong YY, Kang HK (2014) Multimodal imaging evaluation in staging of rectal cancer. World J Gastroenterol 20:4244-4255
97. Heijmen L, Ter Voert EE, Oyen WJ et al (2015) Multimodality imaging to predict response to systemic treatment in patients with advanced colorectal cancer. PLoS One 10:e0120823

98. Goh V, Engledow A, Rodriguez-Justo M et al (2012) The flowmetabolic phenotype of primary colorectal cancer: Assessment by integrated 18F-FDG PET/perfusion CT with histopathologic correlation. J Nucl Med 53:687-692

99. Gu J, Khong PL, Wang S et al (2011) Dynamic contrast-enhanced MRI of primary rectal cancer: Quantitative correlation with positron emission tomography/computed tomography. J Magn Reson Imaging 33:340-347

100. Gu J, Khong PL, Wang S, Chan Q, Law W, Zhang J (2011) Quantitative assessment of diffusion-weighted MR imaging in patients with primary rectal cancer: Correlation with FDG-PET/ CT. Mol Imaging Biol 13:1020-1028

101. Fischer MA, Vrugt B, Alkadhi H, Hahnloser D, Hany TF, VeitHaibach P (2014) Integrated ${ }^{18}$ F-FDG PET/perfusion CT for the monitoring of neoadjuvant chemoradiotherapy in rectal carcinoma: Correlation with histopathology. Eur J Nucl Med Mol Imaging 41:1563-1573 